\title{
Modeling and optimizing IEEE 802.11 DCF for long-distance links
}

\author{
Javier Simó-Reigadas, Andrés Martínez-Fernández, Javier Ramos-López, and Joaquín Seoane-Pascual
}

\begin{abstract}
Most rural areas in developing countries are isolated due to the lack of appropriate low-cost communication technologies. Previous experiences have shown that IEEE 802.11 can be used for the deployment of large static mesh networks with only minor changes to the MAC layer that enable WiFi transceivers to work properly even for very long distances (up to $100 \mathrm{Km}$ in point to point links, and almost $40 \mathrm{Km}$ in point to multipoint setups). However, the impact of distance on performance of such long links has not been deeply analysed. In addition, previous analytical models of IEEE 802.11 DCF can not be applied because they implicitly assume that the propagation time can be neglected. This paper formally studies the impact of the distance on the behaviour of IEEE 802.11 DCF, and presents an analytical model of IEEE 802.11 DCF that accounts for distances correctly. The model is validated with simulations and within a controlled experimental framework, based on wireless channel emulation. Finally, we propose adjustments for ACKTimeout, CTSTimeout, SlotTime, and $C W_{\min }$ parameters that improve significantly the performance of DCF over long distances.
\end{abstract}

Index Terms-IEEE 802.11 DCF, wireless wide-area networks, developing countries.

\section{INTRODUCTION}

$\mathrm{T}$ HERE are extensive rural areas in the world where people have no access at all to communication networks. This is especially true in rural areas of developing countries, where the population is poor and dispersed, and telecom operators work with high costs of deployment and low returns. Some analysts have underlined the strategical role that IEEE 802.11 [1], [2], [3], [4] and VoIP [5], [6] might play for setting up low-cost networks in such scenarios. The IEEE 802.11 standard and its CSMA/CA MAC protocol were specifically conceived for wireless local area networks with maximum distances of hundreds of meters among contiguous stations. Other TDMA-based standards, like IEEE 802.16-2004, are better options for metropolitan or rural broadband wireless networks, at least from a technical point of view. However, 802.11 at present is the only affordable solution in many rural scenarios within developing countries. Additionally experience has proved that long-distance WiFi links are possible with some modification of the MAC layer [7], [8], [9], [10]. This experience suggests that a combination of long-distance point-to-point WiFi links and medium-distance point-to-multipoint infrastructures enables the deployment of low-cost broadband wireless networks in rural areas. Now there is even a generally accepted name for this kind of networks: WiLD (WiFi over Long Distances).

- Javier Simó-Reigadas, Andrés Martínez-Fernández, and Javier RamosLópez are with the Department of Signal Theory and Communications, Universidad Rey Juan Carlos, Camino del Molino s/n, 28943 Fuenlabrada, Spain. E-mail: \{javier.simo,andres.martinez,javier.ramos\}@urjc.es.

- Joaquin Seoane-Pascual is with the Department of Telematics Engineering, Universidad Politécnica de Madrid, Ciudad Universitaria s/n, 28040 Madrid, Spain. E-Mail: joaquin@dit.upm.es.

This work has been partially supported by Research Project TEC2007-68096C02/TCM from Spanish Government.
In the last two years, several scientific publications have partially studied this problem, but only Leung et al. [11] tried to analyze the impact of distance on the behavior of IEEE 802.11 DCF (Distributed Coordination Function). However, their analysis is limited to the feasibility of 802.11 as an alternative for cellular networks. Some authors have assumed implicitly that a CSMA/CA-based MAC is useless for long distances, and have proposed its replacement by a TDMA-based MAC implemented on top of the IEEE 802.11 PHY [12], [13], though a previous try to analyze and optimize the standard MAC has not been addressed. Our group has already demonstrated experimentally [14] that tuning certain parameters of CSMA/CA may yield equivalent performance to that of replacing the protocol. These modifications are easier, cheaper, and compatible with legacy systems. Additionally, many publications propose analytical models of IEEE 802.11 DCF ( [15], [16], [17], [18], [19] among others), but all of them implicitly assume that stations are close to each other. Hence, these models can not be used when stations are several kilometers apart from each other, as later demonstrated in this paper.

Firstly, we present a formal study of how distance between stations affects performance, exploring and identifying causes. Then, a new analytical model of IEEE 802.11 DCF is proposed which incorporates a distance dependency as a fundamental variable. The model has been solved numerically and the results have been compared with experimental long-distance pointto-point links, reproduced under controlled conditions in our laboratory. Several tests have also been made on a real rural WiFi network in Cuzco (Peru) [9] in order to provide a qualitative validation under realistic conditions. The results show that the proposed model captures correctly the behavior of long-distance links. 
Different network simulators have also been used in order to perform a quantitative validation of the pointto-multipoint case, but we have found that none of them capture correctly the effect of the distance on the performance of CSMA/CA, as explained later.

Secondly, the proposed model is used to explain in detail the problems found in real large WiFi networks and to propose the adjustments that optimize their behaviour. Beyond the very basic adjustment of the ACKTimeout and CTSTimeout parameters, it is also known that the slot time must be adapted to optimize the performance of DCF over long distances ${ }^{1}$. We demonstrate how the performance may be optimized by adjusting either the slot time or the $C W_{\min }$ parameter. With the proposed optimization, IEEE 802.11 DCF is shown to perform well in point to point links as long as $100 \mathrm{Km}$, or in point to multipoint links of almost 40 $\mathrm{Km}$ with custom hardware.

The paper is organized as follows. Section II explains the main characteristics of WiLD networks, and Section III briefly introduces the operation of IEEE 802.11 DCF. Section IV examines the maximum allowed distance between stations as supported by the standards. Then, we consider the impact on performance as station separation is extended beyond the maximum distance, and we identify the cause of the performance degradation. Section $\mathrm{V}$ proposes an analytical model of DCF that correctly accounts for the distance. Section VI explains what methods and materials have been used for validating the model, and Section VII analyses the results obtained from the validation. Section VIII proposes several optimization rules for DCF, and finally, Section IX contains the conclusions and future works.

\section{Relevant characteristics of WiLD NETWORKS}

There are quite a few well known examples of IEEE 802.11-based networks in rural areas within developing countries. Experience shows that this technology is often the only one viable in poor remote areas. Some representative examples are [7], [8], [9], [10]. An impressive deployment [20] is the linear network that connects Iquitos in the Peruvian Amazon with several rural health centers, covering a $500 \mathrm{~km}$ stretch along the Napo River up to the country's northern border in 17 hops. Many other examples are not mentioned in scientific papers yet, such as the recent linear network connecting a hospital to the capital city of Malawi along $162 \mathrm{~km}$ in three hops [21].

These WiLD networks usually have a core network in which nodes are interconnected though long point-topoint (PtP) links, and access segments in which access

1. The MadWiFi driver for Linux allows the tuning of three parameters: ACKTimeout, CTSTimeout, and the slot time. The athctrl command accepts a distance as input parameter and modifies them accordingly. However, the values given to slot time are not necessarily optimal, as we will demonstrate in this paper. One can also assign a value directly to any of those parameters within the proc filesystem. points connected to the core network give access to end users through mid-distance point-to-multipoint (PtMP) links. WiFi systems usually have two or more wireless interfaces connected to different external antennas. Those systems replacing the standard MAC protocol [10] tend to avoid interferences among the connected antennas by coordinating transmissions and receptions via different interfaces in the same router. Those using the standard CSMA/CA [20] have to exploit several techniques to avoid interferences, for example, the use of non-overlapping channels together with a sufficient vertical separation between antennas, and orthogonal polarizations.

Regardless of the details of the approach, the final result is a static mesh IP network in which, from the MAC point of view, each PtP or PtMP link is a separate BSS (Basic Service Set) to be analyzed and optimized as an independent network.

\section{A BRIEF INTRODUCTION TO IEEE 802.11 DCF}

The IEEE 802.11 MAC layer defines two coordinating functions: DCF and PCF (Point Coordination Function), but only the first one has been widely implemented in real systems. DCF uses a Carrier Sense Multiple Access protocol with Collision Avoidance (CSMA/CA) that considers all stations as peers (client stations or access points).

The following constant parameters form the basis of DCF:

- Slot time $(\sigma)$ : quantum for defining the contention window time unit and for defining other parameters.

- SIFS (Short Inter Frame Space): Time that separates the end of the reception of a frame and the start of the transmission of its ACK.

- DIFS (Distributed Inter Frame Space): A station needs to sense the channel idle during DIFS seconds before scheduling a new transmission or reactivating the contention window countdown if it was interrupted due to another station's transmission. Its duration depends on the slot time: DIFS = $2 \sigma+S I F S$.

- EIFS (Extended Inter Frame Space): Replaces DIFS when the last transmission that kept the channel busy was corrupted.

A station intending to transmit has to first sense the channel as idle during a DIFS time window. Then, it starts a contention period by calculating a random number of time slots to wait and starts a countdown. The duration of each slot depends on the activity of the channel, this is, idle slots have a fixed duration $\sigma$, but the countdown freezes when the channel is sensed to be busy. This produces a slot that contains a transmission or a collision followed by DIFS or EIFS. When the countdown finishes, the station transmits and starts a timer (ACKTimeout) to wait for an ACK that confirms 
the correct reception. If the ACK is received, the transmission is considered as successful, and the station is ready to restart the whole process again with another frame. If ACKTimeout elapses and no ACK is received, a retransmission is started, following the same process until the maximum retransmission limit is reached. The number of slots in the contention window is a uniform random variable obtained from the distribution $\left[0, C W_{i}\right]$. The range of the distribution, $C W_{i}+1$, grows exponentially each time the transmission is unsuccessful, starting at $C W_{\min }+1$ and ending at $C W_{\max }+1$.

The 802.11 MAC is strongly based in the carrier sense, but has a mechanism to deal with hidden nodes called RTS/CTS (Request to Send / Clear to Send). When activated, a station that is going to transmit a data frame longer than RTSThreshold sends firstly a very short RTS frame (only 14 bytes). If the receiver gets the RTS frame correctly, it sends back a CTS frame, giving the first station the right to transmit the data frame. Both the RTS and the CTS frames contain the information concerning the total foreseen duration of the transaction. This includes all the operations to the point where the channel will again be definitely idle. Stations listening to either the RTS or the CTS frame may initialize an internal timer called NAV (Network Allocation Vector) that causes the same effect to the MAC as the physical carrier detection.

Other details of the MAC operation can be found in the standards [1], [2], [3], and also [22], [23], [24] are recommended for a better understanding.

\section{Distance IMPACTS ON IEEE 802.11 PER- FORMANCE}

The IEEE 802.11 standard is designed to be used over short distances. In this section, we briefly analyze the constraints limiting the maximum distance at which IEEE 802.11 may be used with good performance. For a more extended version of the analysis made in this section, see [25].

\subsection{Limitations Imposed by the PHY}

Any radio link is subject to a link budget: The signal received at one end is equal to the power transmitted at the other end plus the gain of the antennas at both ends minus the attenuation caused by the path loss and the cables and connectors. The radio link is viable only if the received signal power level is greater than the receiver's sensitivity. In fact, a security margin is needed because of the channel variability caused by the weather, the presence of mobile objects, and other factors.

IEEE 802.11 works at ISM non-licensed bands that restrict the maximum transmitted power and the antenna gain. IEEE $802.11,802.11 \mathrm{~b}$ and $802.11 \mathrm{~g}$ work in the $2.4 \mathrm{GHz}$ band, while IEEE 802.11 a works in $5 \mathrm{GHz}$ bands (the specific sub-band depends on the regulatory domain). The regulations in the USA, Japan, and Europe are very different, and countries in other regions may follow different rules, but many developing countries have adopted the FCC (Federal Communications Commission) criteria. FCC permits up to $30 \mathrm{dBm}$ of transmitting power with omnidirectional antennas of gain up to $6 \mathrm{dBi}$ for omnidirectional communications. Directional links may increase the antenna gain with a penalty of $1 \mathrm{dBm}$ for every $1 \mathrm{dBi}$ over $23 \mathrm{dBi}$ for the $5 \mathrm{GHz}$ band, or $1 \mathrm{dBm}$ for every $3 \mathrm{dBi}$ over $6 \mathrm{dBi}$ for the $2.4 \mathrm{GHz}$ band.

The transmitting power restriction is a limiting factor in terms of distance. We have calculated approximately the distances that can be achieved with typical values of transmitted power, sensitivity, antenna gain, and cable attenuation ${ }^{2}$. The propagation loss has to be estimated for each specific environment, as irregular terrains condition has a considerable effect on the propagation. However, a rough estimation of achievable distances obtained by using the free space model is presented in Fig. 1. A stability margin of $20 \mathrm{~dB}$ has been preserved over the sensitivity for calculating the minimum acceptable received power level. We have calculated the maximum achievable distance for PtP links (both ends with directional antennas), PtMP links (one end directional, the other end omnidirectional), and mesh networks with omnidirectional antennas in all nodes.

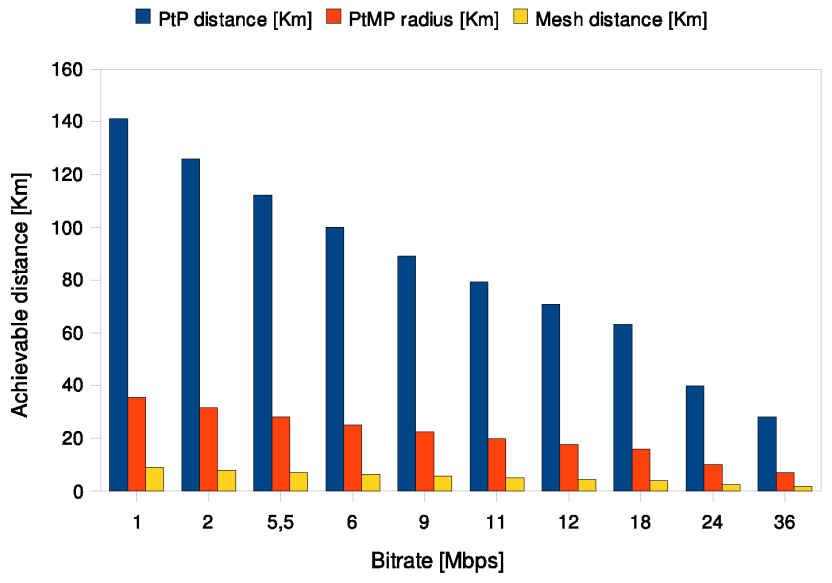

Fig. 1. Achievable distances for different bit rates under FCC 15.247 regulation for the $2.4 \mathrm{GHz}$ band, depending on the gain of antennas at both ends.

The coverage within the $5 \mathrm{GHz}$ band is similar because the propagation losses are higher at those frequencies, but better antenna gains compensate for this effect. In real networks, such as the one presented in [9], the link budgets correspond with these approximations. We can see that very long links are feasible while still respecting the power restrictions, specially with the most robust (slowest) modulations. Only concerning the PHY, PtP links as long as $140 \mathrm{Km}$ are possible. In the case of PtMP setups, a radius up to $35 \mathrm{Km}$ is achievable. Mesh

2. Specifications taken from the datasheets of Ubiquity XR2 miniPCI cards, Hyperlink $12 \mathrm{dBi}$ dipole antennas and $24 \mathrm{dBi}$ grid antennas, normal u.FL-to-N pigtails and $3 \mathrm{GHz}$ outdoor coaxial cables 
networks in which nodes have omnidirectional antennas would admit maximum distances between contiguous nodes of $9 \mathrm{Km}$ or less. Beyond the power limitations and the derived LOS (line of sight) requirement, the PHY does not contain any other restrictive aspects regarding the distance.

\subsection{MAC Layer Imposed Bounds}

The description of DCF in the IEEE 802.11 standard family [1], [2], [3], [4] does not mention distances or distance limits, but it accounts for the distance implicitly wherever the propagation time is considered. However, the standard assumes that the propagation time itself (defined by the AirPropagationTime variable) has a maximum value of $1 \mu \mathrm{s}$. Also the slot time, which is the only MAC parameter that accounts for the propagation time at a higher level, is also a PHY-dependent constant. Other parameters depend in turn on SlotTime (ACKTimeout, DIFS). In other words, the standard expects DCF to be used within a range of as much as some hundreds of meters. The original standards [1], [2] did not define accurately the meaning of the AirPropagationTime variable, but the last revision of the standard [4] states precisely that it is "twice the propagation time (in microseconds) for a signal to cross the maximum distance between the most distant allowable STAs that are slot synchronized". It must be highlighted that the virtual carrier mechanism neglects the propagation time, which causes the NAV to be inaccurate when distances are longer than expected. Hence, the following elements need to be studied: (1) ACKtimeout \& CTSTimeout, (2) the slot time, (3) the computation of the $N A V$ for the virtual carrier detection, (4) the Coverage Class, and (5) the DIFS. Each of these key elements are considered next.

\subsubsection{ACKTimeout \& CTSTimeout}

In basic mode, when ACKTimeout is too short for a given distance, ACK frames arrive systematically too late at the waiting station. If this happens, all ACK frames are discarded and the standard indicates that every data frame is retransmitted ShortRetryLimit times before being dropped (default is 7 retransmissions). This behavior is illustrated in Fig. 2. If this happens, several copies of each packet arrive to destination correctly even if the transmitter does not know it. The link works because data frames are in fact being received at the destination, but it exhibits a very poor performance.

In RTS/CTS mode, the sender node waits for a duration of the CTStimeout interval for the returned CTS frames, which does not arrive due to propagation delays. The cycle is repeated up to LongRetryLimit times (default value is 4 times). In this case the link does not work because data frames never pass through.

The value of the ACKTimeout parameter was not clearly established in the text of the original standard. Some authors [2], [23]) have interpreted that the MAC formal description fixes the value of the

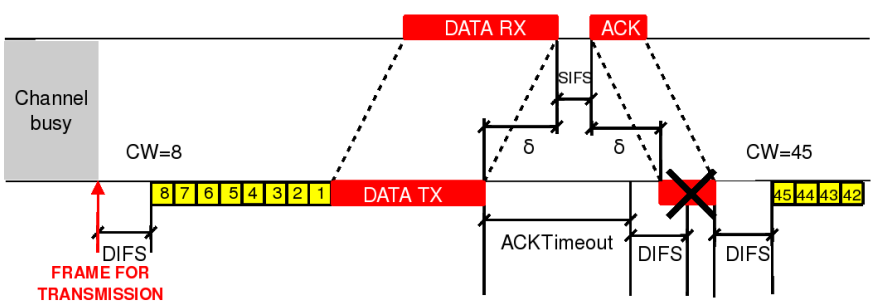

Fig. 2. Systematic loss of ACK frames due to a distance exceeding the limit imposed by the ACKTimeout value.

$A C K$ Timeout $=S I F S+\sigma_{S T D}+A C K+P L C P$, where $\sigma_{S T D}=$ Standard SlotTime, PLCP is the total duration of the preamble plus the physical layer header, and ACK is the duration of the ACK MAC frame. Leung et al. [11] affirm that the value of ACKTimeout is not given in the standard. However, the last revision of the standard [4] clearly states that

$$
A C \text { KTimeout }=S I F S+\sigma_{S T D}+P L C P
$$

According to the standard, an aPHY-RXSTARTIndication has to be passed to the MAC layer before the ACKTimeout expires, or the ACK will be discarded. The SIFS time is consumed at the receiver side, and the PLCP time is required for the transmitter's PHY to pass the aPHY-RX-START-Indication after the first bit of the ACK preamble is received. Hence, the slot time is in the sum because conceptually it contains the roundtrip propagation time and enough time for the CCA (Clear Channel Assessment) mechanism. The round-trip propagation time is expected by the standard to last as much as $1 \mu \mathrm{s}$ (only enough for $150 \mathrm{~m}$ between stations), but the CCA leaves a minimum margin of $5 \mu \mathrm{s}$ in the slot time (enough for $750 \mathrm{~m}$ ). For implementations of the MAC yielding a faster CCA, the time left for propagation has to be longer.

For distances longer than $150 \mathrm{~m}$, we guarantee that ACKTimeout has a sufficient value by defining it as

$$
A C \text { KTimeout }=\text { SIFS }+\sigma_{S T D}+2 \delta_{M A X}+P L C P
$$

for long-distance 802.11 links, where $\delta_{M A X}$ is the maximum propagation time in the BSS. As seen above, it has been unclear for years what the value of ACKTimeout should be. We have checked real hardware in a controlled environment to determine how real cards implement the ACKTimeout. A hardware channel emulator Elektrobit PropSim C8 was used for this purpose (Figure 7). Two embedded computers running Voyage Linux with IEEE 802.11 cards were introduced in RF shielded boxes and connected to each other through the emulator. We compared cards based on the two dominant chipsets for long range WiFi installations: Intersil Prism 2.5 and Atheros AR5212. For cards based on the first chipset, ACKTimeout is internally fixed and does not match the standard value, permitting links as long as 
23,5 $\mathrm{Km}$ for $2 \mathrm{Mbps}$ bit rate. On the other hand, cards based on the Atheros chipset permit the adjustment of the ACKTimeout parameter. We were able to adjust ACKTimeout to permit links as long as $105 \mathrm{Km}$. Higher values of ACKTimeout were not possible due to hardware constraints ${ }^{3}$.

ACKTimeout tests were also run in two network simulators: OPNET Modeler 11.5 and NS-2.30. Fig. 3 represents an NS-2 simulation showing the throughput between two stations decreasing slowly as the distance increases, and that there is a sharp decrease when the distance passes the ACKTimeout limit. The same behavior has been found in all hardware devices and software simulators, but the sharp decrease occurs at different distances in all cases. At $2 \mathrm{Mbps}$ of bitrate, the default maximum distance was $5.8 \mathrm{Km}$ for Atheros chipsets, $23.5 \mathrm{Km}$ for Prism 2.5 chipsets (fixed), $600 \mathrm{~m}$ for NS-2 (can be changed in the code), and $45 \mathrm{Km}$ for OPNET simulator (fixed). For further experiments, we developed a patch for NS-2.30 that makes it possible to set the DSSS_MaxPropagationDelay value at simulation time (which in turn is used internally to set the ACKTimeout) [26].

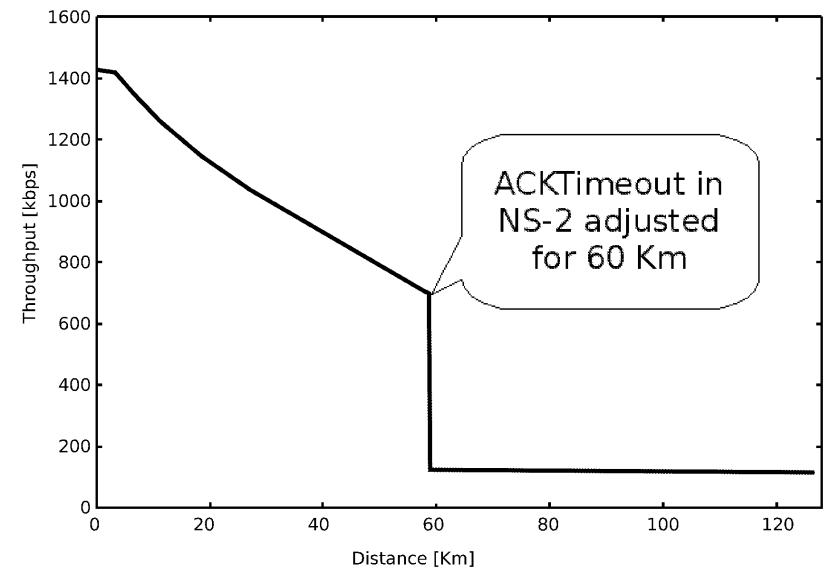

Fig. 3. Rate versus distance for an FTP file transfer simulated with NS-2.30 with DSSS $S_{M}$ axPropagationDelay = $400 \mu s$.

For the rest of this paper, ACKTimeout is always supposed to be modified as proposed in Equation 2.

\subsubsection{The slot time}

The slot time is a PHY-dependent constant. For example, it is $20 \mu \mathrm{s}$ for $802.11 \mathrm{~b}$ and $9 \mu \mathrm{s}$ for $802.11 \mathrm{a}$ and pure $802.11 \mathrm{~g}$ networks. These values have been estimated accounting for the time required for the CCA mechanism, the $\mathrm{Rx} / \mathrm{Tx}$ turnaround time, the propagation time, and

3. MadWifi permits us to adjust ACKTimeout directly through the Linux proc-filesystem, or indirectly through the athctrl command. However, that number is stored in a hardware register that limits its value to $744 \mu \mathrm{s}$ for $802.11 \mathrm{~b}$, to $372 \mu \mathrm{s}$ for $802.11 \mathrm{~g}$ and to $409 \mu \mathrm{s}$ for 802.11a. Those values limit the range at the MAC level to 105, 49 and $55 \mathrm{Km}$ respectively. the MAC processing delay. Some cards actually allow to adjust the slot time, but the basic standard [1] does not allow users to do so. In the following, we analyse the impact of keeping the slot time at its standard value for any distance.

The principle underlying the slot time is that two stations intending to transmit will collide if, and only if, they start the transmission in the same time slot. If both transmissions are scheduled for different slots, the second transmitting station will have the time to detect the first transmission and will freeze and backoff, thus avoiding the collision. Obviously, this behaviour requires that the propagation time plus the CCA time is smaller than $\sigma$.

Let us define the Vulnerability Interval (VI) between two stations as the time interval during which the frame transmitted by one station could collide with the frame transmitted by another one at a given time. Let us define also $\delta_{Q D}=\delta_{D Q}$ as the propagation delay between stations $E_{Q}$ and $E_{D}$. Note that a transmission is considered successful by the transmitter if it receives a correct ACK from the receiver. Let us define CCA as the time required by the receiver to perform the CCA function.

Consider the following scenario with three stations $E_{Q}, E_{D}$ and $E_{X}$ mutually visible. Station $E_{Q}$ transmits a packet to $E_{D}$, who answers with the corresponding ACK. Station $E_{X}$ will try to transmit at the same time, possibly producing a collision. In general, if $E_{Q}$ transmits at time instant $t, E_{X}$ will only produce a collision if it starts its transmission during the vulnerability interval $V I_{Q, X}=\left(t-\delta_{Q, X}-C C A, t+\delta_{Q, X}+C C A\right)$. Otherwise, either $E_{Q}$ does not transmit since it has detected a transmission from $E_{X}$, or $E_{X}$ does not transmit since it has detected a transmission from $E_{Q}$. However, the impact on the performance will depend on the number of slots starting in this $V I_{Q, X}$ interval. We define the normalized vulnerability interval $\left(N V I=\frac{V I}{\sigma}\right)$, whose value depends on the distance. Let us consider the following cases, represented in Fig. 4:

1) $E_{X}$ is very close to $E_{Q}$ so that $\delta_{Q, X}+C C A<\frac{\sigma}{2}$. The collision takes place only if $E_{X}$ starts the transmission in the same slot as $E_{Q}$, so $N V I_{Q, X}=1$. Cases a) and b) in Fig. 4 both match with this condition and differ in the importance of the propagation time compared to $\sigma$.

2) $E_{X}$ is far from $E_{Q}$ so that $\delta_{Q, X}+C C A>\frac{\sigma}{2}$. Two or more slots can start now during the $V I_{Q, X}$. If $E_{X}$ begins a transmission in any of them, a collision will take place. Note that the Bianchi model [23] or others derived from it are no longer valid in this case, so we can establish a constraint of the form $2 \delta_{Q, X}+2 \cdot C C A \leq \sigma$ for these models. For $802.11 \mathrm{~b}$, where the slot time is $20 \mu \mathrm{s}$, this constraint may be approximately between $750 \mathrm{~m}$ and $2,5 \mathrm{Km}$, depending on the effective CCA time. For $802.11 \mathrm{a} / \mathrm{g}$ those values are $9 \mu \mathrm{s}$ and $750 \mathrm{~m}-1.1 \mathrm{Km}$, respectively. The CCA time will be neglected in longdistance analysis because, as the distance grows, 

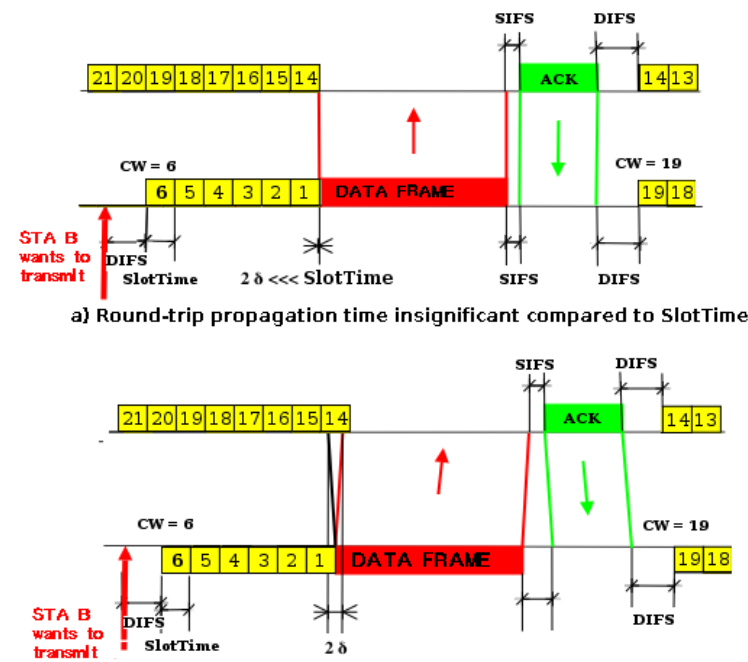

b) Round-trip propagation time significant but smaller than SlotTime

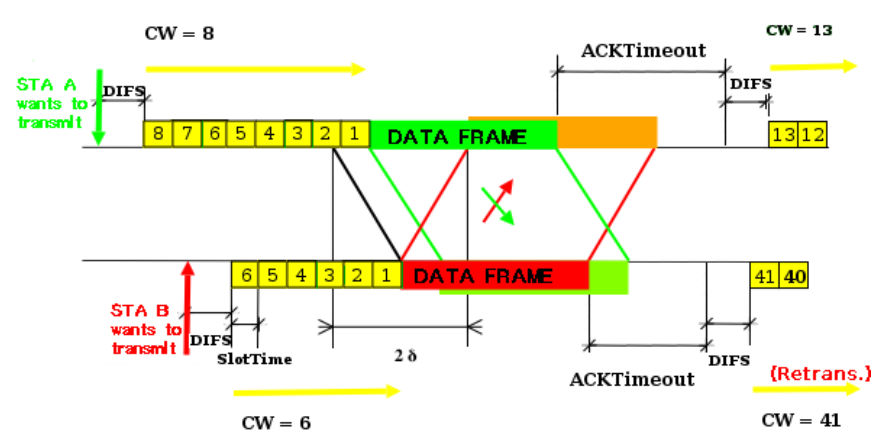

c) Round-trip propagation time is greater than SlotTime

Fig. 4. Behaviour of the MAC in two contending stations depending on the distances between them.

$\delta_{Q X} \gg C C A$. Under these conditions, we can write $N V I_{Q, X}=\frac{2 \delta_{Q, X}}{\sigma}$. The non-integer decimal part of $N V I$ corresponds to the probability that an extra slot beginning in the vulnerability interval, i.e., the number of slots beginning in the vulnerability interval is at least $\operatorname{int}\left(\frac{2 \delta_{Q, X}}{\sigma}\right)$ and the decimal part of the fraction is the probability there is one more slot or int $\left(\frac{2 \delta_{Q, X}}{\sigma}\right)+1$ slots beginning in $V I_{Q, X}$.

If the slot time is not considered as constant, then the $V I_{Q, X}$ can always be minimized.

\subsubsection{DIFS}

The different inter-frame spaces are defined to assign different priorities to ACK frames, PCF control frames and normal DCF data frames. In a DCF network, the main role of DIFS is to prevent stations waiting for the channel from colliding with ACK frames. As DIFS $=S I F S+2 \sigma$, Leung et al. [11] interpret that this marks a distance limit corresponding to the round-trip propagation time of approximately $2 \sigma$. In fact, the distance limit would be longer because the DurationID field in the data frame header, which is used to set the NAV for the virtual carrier sense mechanism, also accounts for the expected
ACK frame. However, the adaptation of the slot time, which is contained twice in the DIFS, would adapt this limit to a longer distance if required.

\subsubsection{NAV Computation}

Until now, it has been assumed that all the stations are able to listen to the others. However, this is unlikely to happen where long distances and directive antennas are considered. When considering hidden nodes, the standard offers the RTS/CTS mode, so that nodes that cannot listen to any of the stations participating in a communication may know how long the channel is going to be busy by using the virtual carrier detection, which in turn is based on the information in the DurationID field contained either in the RTS or in the CTS packet. The standard accurately defines the way this time is computed, but it does not take the propagation time into consideration which produces an anomalous behaviour when long distance links are used. The magnitude of those inaccuracies depends greatly on the distances and visibilities among the three stations involved [25].

If propagation times are long, stations receiving the RTS will receive the data frame much later than expected, but they would not transmit during that time in a real case because the channel is still reserved for the transmission of the data frame and the following ACK. The NAV is updated upon data frame reception, and then the situation is the one presented in the previous point. On the other hand, stations receiving the CTS but not the RTS could produce a collision until they receive the CTS, but once they receive it, they will receive the ACK correctly unless the round-trip propagation time is longer than $A C K+D I F S$, which is unlikely in a real situation. If visibility between nodes is not symmetrical, other more complex situations could occur.

As a result, the probability of collision with hidden nodes increases even if RTS/CTS is used, making this mode useless. If the slot time is adapted to include the round-trip propagation time, then DIFS includes twice the round-trip propagation time and RTS/CTS transactions are partially protected against collision from hidden nodes, with the only adverse effect of possible early accesses to the channel by those nodes after the ACK.

\subsubsection{Coverage Class}

The recent revision of the standard [4] explicitly considers that 802.11 may be used for metropolitan area networks and introduces a regulatory triplet, considering aspects such as the transmission power limit and the coverage class. It is stated that the slot time may be altered through a Coverage Class parameter. If this optional feature is activated, the Coverage Class parameter gets an integer value in the range $[0,31]$ and then the standard slot time gets increased in 3 . CoverageClass microseconds.This permits us to adapt DCF to BSS with a maximum radius of $15 \mathrm{Km}$, as it changes directly the slot time as required for minimizing the vulnerability 
interval, and changes indirectly the ACKTimeout. Longer distances are not supported by Coverage Class feature.

\subsection{The Distance in Previous DCF Analytical Models}

A number of papers propose analytical models of IEEE 802.11 DCF, most of them based on the seminal paper by Bianchi [16] of a model using a bi-dimensional Markov chain. The model was based on some strong simplifications, such as the limited number of retransmissions, the constant transmission probability and conditional collision probability for all stations and all stages, and the complete visibility among stations. For the rest, the model captured all the complexity of DCF, hence giving very good results. After this model, many other authors published their own analysis on improving or extending Bianchi's bi-dimensional Markov chain. In [18], [27], [28], the authors took into account the finite number of retransmissions. Other models included variable packet sizes [29], or introduced the effects of non-ideal wireless channels [19], among other extensions. In 2005, Bianchi and Tinnirello proposed an alternative model only based on conditional probability, incorporating the corrections and contributions from other authors to Bianchi's model [17].

There are also a number of authors that have proposed alternative models that are not based on Bianchi's approach. None of the previous models can be applied to the case of long distances among stations, but [17] has some valuable aspects that have to be taken into account in this work.

Bianchi and Tinnirello [17] defined a model slot as the interval between two consecutive decrements of the contention window in a non-transmitting station. A station with counter at value $b$ at a given slot will certainly transmit $b$ slots later. The slot duration depends on what the slot contains, and hence, it can be empty, contain a collision, or contain one or several successful transmissions. There is a non-null probability that a station having transmitted a frame successfully gets a contention window of zero slots for the next transmission and wins the channel again before the other stations decrement their counters. Due to this behaviour, Bianchi and Tinnirello calculate effective values for some parameters accounting for the probability that a slot contains more than one successful transmission, as follows:

$$
E[P]^{\prime}=E[P]+\sum_{k=1}^{\infty} B_{0}^{k} E[P]=\frac{E[P]}{1-B_{0}}
$$

where $E[P]^{\prime}$ is the effective average packet size, $P$ is the packet size, and $B_{0}=\frac{1}{C W_{\min }+1}$. The average duration of a successful transmission and that of a collision are redefined in the same way:

$$
\begin{gathered}
T_{s}^{\prime}=T_{s}+\sum_{k=1}^{\infty} B_{0}^{k} T_{s}=\frac{T_{s}}{1-B_{0}} \\
T_{c}^{\prime}=T_{c}+\sigma
\end{gathered}
$$

These equations will be used later in this paper. The definition of the contention window in [17] is mathematically different from previous models. Now, $W_{i}=$ $\min \left(2^{i}\left(C W_{\min }+1\right)-1, C W \max \right)$ for $i=(1, \ldots, R)$, but $W_{0}=C W_{\min }-1$ because of the new definition of a model slot.

In these terms, Bianchi and Tinnirello use conditional probabilities to obtain a set of two equations with two unknowns. The unknowns are the transmission probability at any slot $(\tau)$ and the conditional collisional probability $p$ for a transmission for a starting transmission, yielding the following expressions:

$$
\begin{gathered}
\tau=\frac{1}{1+\frac{1-p}{1-p^{R+1}} \sum_{i=0}^{R} p^{i}\left(1+\frac{C W_{i}}{2}\right)} \\
p=1-(1-\tau)^{n-1}
\end{gathered}
$$

Equation 6 represents the internal behaviour of DCF for any given station, while Equation 7 represents the interaction among stations. It is considered that all stations have the same probabilities $\tau$ and $p$ and have the same perception of the network. It is obvious that Equation 6 is true for each station, no matter what the inter-station distances are, while Equation 7 assumes a symmetry that is not necessarily true for wide area networks with more than two nodes.

Equation 7 also assumes that two stations only collide when transmitting in the same slot, which is not true for long distances, as seen in the previous section. For any given station $E_{Q}$, the transmission probability in a given slot is $\tau$, so $(1-\tau)$ is the probability of that station not transmitting in any given slot, $(1-\tau)^{n-1}$ is the probability of all other $(n-1)$ stations not transmitting in the same given slot (given that all have the same transmission probability $\tau$, which is not necessarily true), and $1-(1-\tau)^{n-1}$ would be the probability of at least one of them transmitting at a given slot. As $p$ is defined as the conditional probability of colliding once a packet is going to be transmitted in a given slot, Equation 7 implies that two transmissions can collide only if they start in the same slot, which is only true for short propagation times. As a consequence, [17] provides a part of a DCF analytical model that is independent of distances, and a new analysis is required in order to complete that model.

\section{A NEW ANALYTICAL MOdEL OF IEEE 802.11 DCF FOR LONG DISTANCES}

In this section we develop an analytical model of IEEE 802.11 DCF that takes into account intrinsically the propagation times among stations.

\subsection{Assumptions}

The following assumptions are made:

H1.- No hidden nodes will be considered. In real longdistance WiFi networks, there are hidden nodes 
in almost all cases, so this could seem a limiting assumption. However, a significant part of the performance problems of these networks are present even if there are not any hidden nodes, and may be better studied and explained with this assumption. The extreme complexity introduced by hidden nodes will be analysed in a future work.

H2.- The channel is ideal and frames are always acknowledged unless they collide. Also, ideal channels do not exist, but real channels may have almost ideal behaviour, if the received signal is strong enough (which may be a design criteria). Dong and Varaiya [19], as well as other authors, show how the BER (Bit Error Rate) can be easily taken into account if necessary just by redefining $p$ as the probability of a packet that is going to be transmitted in a given slot to be lost, either due to a collision or due to transmission errors.

H3.- Every station always has a frame that is ready for transmission. Also, the saturation condition is not real, but gives a worst-case condition.

H4.- The conditional collision probability $p_{Q}$ is constant and stage independent for station $E_{Q}$. This assumption is known to be good enough because it is common in analytical models and does not depend on the distances at all. On the other hand, we have also studied analytically the consequences of eliminating this assumption, and in this case, the model quickly becomes unmanageable.

H5.- The probability that a transmission starts and finishes in any single vulnerability interval $V I_{Q D X}$ of the network can be neglected. In other words, the shortest possible transmission is longer than the longest vulnerability interval in the network.

H6.- Distances among stations can be as long as 100 $\mathrm{Km}$. ACKTimeout is supposed to be adjusted as required for each distance.

\subsection{Model for $\mathrm{n}$ Active Stations Without Hidden Nodes}

Let us consider $n$ stations $E_{1}, \ldots, E_{n}$ with full visibility among them. Any two stations $E_{Q}$ and $E_{D}$ are separated $d_{Q D}$ meters, and the propagation time between them is $\delta_{Q D}=\delta_{D Q}=\frac{d_{D Q}}{c}$.

For station $E_{Q}, p_{Q}$ is the conditional collision probability, and $\tau_{Q}$ is the transmission probability at any slot. Each station $E_{Q}$ sees the network differently, thus having its own probabilities $\tau_{Q}$ and $p_{Q}$ and perceiving time slots at very different instants. Hence, for the $n$ stations we have $2 n$ different variables $\left(p_{1}, \ldots, p_{n}, \tau_{1}, \ldots, \tau_{n}\right)$.

As explained before, Equation 6 is true for each station as long as H1, H2, H3, and H4 are satisfied. So, we may define a set of $n$ equations as (8) with $2 n$ unknowns for stations $E_{Q}$, where $Q \in(1, \ldots, n)$ :

$$
\tau_{Q}=\frac{1}{1+\frac{1-p_{Q}}{1-p_{Q}^{R+1}} \sum_{i=0}^{R} p_{Q}^{i}\left(1+\frac{W_{i}}{2}\right)}
$$

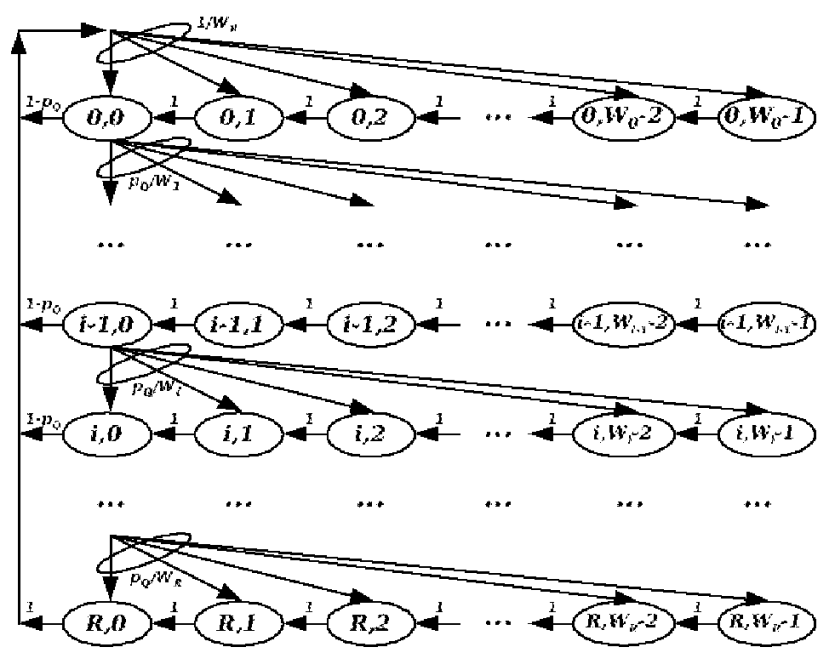

Fig. 5. Model of DCF with a bi-dimensional Markov chain compatible with Bianchi and Tinnirello's model.

where $W_{i}=\min \left(2^{i}\left(C W_{\min }+1\right), C W_{\max }+1\right)$ for $i=(1$, $\ldots, R)$, and $W_{0}=C W_{\text {min }}$.

We need $n$ additional equations to complete a system with the $2 n$ unknowns. The effect of the distance must be considered in depth for this purpose. Let us define $\xi_{Q D X}$ as the probability that station $E_{X}$ collides with a transmission from $E_{Q}$ to $E_{D}$. Such collision will happen if $E_{X}$ starts a transmission during the corresponding vulnerability interval $V I_{Q D X}$. Let us also define $\mu_{Q D}$ as the probability that a transmission from station $E_{Q}$ is destined for $E_{D}$. In the particular case that all possible destinations have always the same probability, then $\mu_{Q D}=\frac{1}{n-1}$.

The probability that a transmission from $E_{Q}$ collides may be expressed as

$$
p_{Q}=\sum_{D=1, D \neq Q}^{n} \mu_{Q D}\left(1-\prod_{X=1, X \neq Q}^{n}\left(1-\xi_{Q D X}\right)\right)
$$

To obtain a convenient expression for $\xi_{Q D X}$, we need to calculate the steady state probabilities of a station to be in any model slot as defined previously. Those probabilities can be calculated by defining a bi-dimensional Markov chain for each station $E_{Q}$ that must be compatible with the assumptions and definitions given for the first part of the model, and must share the same definition of a slot and consider the same definition of $W_{i}, i \in[0, R]$. Such a Markov chain is shown in Fig. 5, and is almost identical to the Markov chain proposed by Chatzimisios et al. in [18]. Equation (8) can also be derived from it.

This chain models the bi-dimensional discrete-time stochastic process $b_{Q}[n], s_{Q}[n]$ where $b_{Q}[n]$ and $s_{Q}[n]$ represent the backoff timer and the backoff stage respectively for station $E_{Q}$ at slot n. Let 


$$
b_{Q, i, k}=\lim _{n \rightarrow \infty} P\{s[n]=i, b[n]=k\}
$$

be the stationary distribution of the Markov chain, where $i \in[0, R]$, and $k \in\left[0, W_{i}-1\right]$. When station $E_{X}$ is at stage $i$ ( $i^{\text {th }}$ retransmission) and state $j$ (it will transmit within $j$ slots), it is said to be at $e_{X, i, j}$.

There are two minor differences between this Markov chain and the one in [18]:

- The conditional probability of collision is now specific for each station, and accordingly, the nomenclature has changed using $p_{Q}$ instead of $p$.

- The fist stage now has $W_{0}=C W_{\min }$ as explained above, instead of $W_{0}=C W_{\min }+1$ as for Chatzimisios et al.

However, similar relationships as in [18] may be obtained:

$$
\begin{aligned}
b_{Q, i, 0} & =p_{Q}^{i} b_{Q, 0,0} \quad, i \in[0, R] \\
b_{Q, i, k} & =\frac{W_{i}-k}{W_{i}} b_{Q, i, 0}, i \in[0, R], k \in\left[0, W_{i}-1\right] \\
b_{Q, 0,0} & =\tau_{Q} \frac{1-p_{Q}}{1-p_{Q}^{R+1}}
\end{aligned}
$$

Let us define the events $\left\{A_{Q, D, X, j}\right\}=$ $\left\{E_{X}\right.$ transits from $e_{X, i, j}$ to $e_{X, i, 0}$ during $\left.V I_{Q D X}\right\}$ and $\left\{B_{X, j}\right\}=\left\{E_{X}\right.$ receives nothing in $j$ consecutive slots $\}$. The first could also be defined as $\left\{A_{Q, D, X, j}\right\}=$ $\left\{V I_{Q D X}\right.$ contains at least $j+1$ starting slots of $\left.E_{X}\right\}$.

The calculation of $\xi_{Q D X}$ is strongly based on assumption H5. We will consider that $E_{X}$ can only collide with $E_{Q}$ if it does not receive another frame during the vulnerability interval and before its transmission. Then, $\xi_{Q D X}$ could be calculated as the sum of all $b_{X, i, j}$, each multiplied by the probability for $E_{X}$ to get from $e_{X, i, j}$ to transmission state before a time $V I_{Q D X}$ elapses. This is expressed as

$$
\begin{aligned}
\xi_{Q D X} & =\sum_{i=0}^{R} \sum_{j=0}^{C W_{i}} b_{X, i, j} P\left\{A_{Q, D, X, j}\right\} \\
& =\tau_{X}+\sum_{i=0}^{R} \sum_{j=1}^{C W_{i}} b_{X, i, j} P\left\{A_{Q, D, X, j}\right\}
\end{aligned}
$$

If distances are short and stations only collide when they transmit in the same slot, the only non-null terms are for $j=0$, and then $\xi_{Q D X}=\tau_{X}$ because $\tau_{X}=$ $\sum_{i=0}^{R} b_{X, i, 0}$. Also the saturation condition implies for short distances that $\mu_{X Q}=\frac{1}{n-1}, \forall E_{X}, E_{Q}$. In this case, it is straight forward to see that (9) reduces to (7). Applying the Bayes' Theorem

$$
P\left\{A_{Q, D, X, j}\right\}=\frac{P\left\{A_{Q, D, X, j} \mid B_{X, j}\right\} \cdot P\left\{B_{X, j}\right\}}{P\left\{B_{X, j} \mid A_{Q, D, X, j}\right\}}
$$

and considering that $P\left\{B_{X, j} \mid A_{Q, D, X, j}\right\}=1$ due to assumption $\mathrm{H} 5$, we may have $\xi_{Q D X}$ by calculating $P\left\{A_{Q, D, X, j} \mid B_{X, j}\right\}$ and $P\left\{B_{X, j}\right\}$.
The first of those probabilities will be called $K_{Q D X, j}=$ $P\left\{A_{Q, D, X, j} \mid B_{X, j}\right\}$ in the next equations, and is defined by

$$
K_{Q D X, j}= \begin{cases}1 & \operatorname{int}\left(N V I_{Q D X}\right)>j \\ \left(N V I_{Q D X}-j\right) & \operatorname{int}\left(N V I_{Q D X}\right)=j \\ 0 & \operatorname{int}\left(N V I_{Q D X}\right)<j\end{cases}
$$

The effect of multiplying a term by $K_{Q D X, j}$ has the effect of preserving it if the vulnerability interval contains more than $j$ complete empty slots, to annulate it if the vulnerability interval never contains $j$ starting slots, and to multiply the term by the probability of containing exactly $j$ starting slots in the intermediate case.

Regarding the second probability defined above, neglecting the previous history of frame exchanges in the network, $P\left\{B_{X, j}\right\}$ is the product of two independent probabilities:

1) Probability that stations different from $E_{X}$ and $E_{Q}$ do not transmit during a time $j \sigma$, so that all of them must still transit through $j$ slots before transmitting.

2) Probability that $E_{X}$ gets a contention window higher than $j$, no matter in what stage it is.

Based on this, we define

$$
\begin{aligned}
P\left\{B_{X, j}\right\}= & \left(1-\mu_{X Q} \sum_{a=0}^{R} \sum_{b=0}^{W_{a}-1} \min \left(\frac{j}{W_{a}}, 1\right) b_{X, a, b}\right) \\
& \prod_{y=1, y \neq X, Q}^{n} \sum_{l=0}^{R} \sum_{m=j}^{W_{l}-1} b_{y, l, m}
\end{aligned}
$$

Replacing in (15) and then in (14), we obtain (18).

We can replace variables $b_{y, l, m}$ in (18) using their definition in (13), and then we have (19) that represents $n$ equations $\forall Q \in(1, \ldots, n)$ with $2 n$ unknowns, thus completing the set of $2 n$ equations with $2 n$ unknowns of our model.

\subsection{Performance calculation}

The total throughput can be calculated as

$$
S=\sum_{i=1}^{n} S_{i}
$$

where $S_{i}$ is defined as the average number of bits transmitted per slot divided by the average slot size,

$$
S_{i}=\tau_{i} \frac{\left(1-p_{i}\right) E[P]^{\prime}}{E[S l o t]}
$$

The average slot duration is calculated taking into account the different contents that a station $E_{i}$ can perceive in a slot. A slot can be empty, busy by a transmission from $E_{i}$, busy by another station's transmission, busy by a collision that involves $E_{i}$ or busy by a collision that does not involve $E_{i}$, this is, 


$$
\xi_{Q D X}=\sum_{i=0}^{R} \sum_{j=0}^{W_{i}-1} K_{Q D X, j} b_{X, i, j}\left(\prod_{y=1, y \neq X, Q}^{n} \sum_{l=0}^{R} \sum_{m=j}^{W_{l}-1} b_{y, l, m}\right)\left(1-\mu_{X Q} \sum_{a=0}^{R} \sum_{b=0}^{W_{a}-1} \min \left(\frac{j}{W_{a}}, 1\right) \cdot b_{X, a, b}\right)
$$

$$
\begin{aligned}
p_{Q}= & \sum_{D=1, D \neq Q}^{n} \mu_{Q D}\left\{1-\prod_{X=1, X \neq Q}^{n}\left[1-\sum_{i=0}^{R} \sum_{j=0}^{W_{i}-1} K_{Q D X, j} \frac{W_{i}-j}{W_{i}} p_{X}^{i} \tau_{X} \frac{1-p_{X}}{1-p_{X}^{R+1}}\right.\right. \\
& \left.\left.\left(\prod_{y=1, y \neq X, Q}^{n} \sum_{l=0}^{R} \sum_{m=j}^{W_{l}-1} \frac{W_{l}-m}{W_{l}} p_{y}^{l} \tau_{y} \frac{1-p_{y}}{1-p_{y}^{R+1}}\right)\left(1-\mu_{X Q} \sum_{a=0}^{R} \sum_{b=0}^{W_{a}-1} \min \left(\frac{j}{W_{a}}, 1\right) \frac{W_{a}-b}{W_{a}} p_{X}^{a} \tau_{X} \frac{1-p_{X}}{1-p_{X}^{R+1}}\right)\right]\right\}
\end{aligned}
$$

$$
\begin{aligned}
E\left[\text { Slot }_{i}\right]= & \left(1-P_{t r}\right) \sigma+\sum_{j=1}^{n} \tau_{j}\left(1-p_{j}\right) T_{s, j, i}+ \\
& P_{t r}\left(1-P_{s}\right)\left(\frac{\tau_{i}}{P_{t r}} T_{c, i}+\left(1-\frac{\tau_{i}}{P_{t r}}\right) T_{c, n o t-i}\right)
\end{aligned}
$$

where

$$
P_{t r}=1-\prod_{x=1}^{n}\left(1-\tau_{x}\right)
$$

is the probability of a slot to be busy by any transmission,

$$
P_{t r} P_{s}=\sum_{x=1}^{n} \tau_{x}\left(1-p_{x}\right)
$$

is the probability of successful transmission,

$$
T_{s, j, i}=\frac{1}{1-B_{0}} \begin{cases}T_{s, \text { short }} & i \neq j \\ T_{s, \text { short }}+2 \cdot E\left[\delta_{i}\right] & i=j\end{cases}
$$

is the duration of a successful transmission. In (25), $B_{0}=$ $\frac{1}{C W_{\min }+1}$ was already defined in (3),

$$
T_{s, \text { short }}=E[P]+2 \cdot P L C P+S I F S+A C K+D I F S
$$

is the average duration of a successful transmission in the case of neglected propagation time,

$$
T_{c, i}=\sigma+E[P]+P L C P+A C K \text { Timeout }+ \text { DIFS }
$$

is the duration of a collision that involves to station $E_{i}$, and

$$
T_{c, n o t-i}=\sigma+E[P]+P L C P+E I F S
$$

is the duration of a collision that does not involve to station $E_{i}$.

The intuitive values of successful transmission time and collision time are corrected as in (3) and (4) due to the definition of a model slot, as explained before. The average propagation time needed for $T_{s, j, i}$ is defined as

$$
E\left[\delta_{i}\right]=\sum_{j=1, j \neq i}^{n} \mu_{i, j} \delta_{i, j}
$$

The packet dropping probability is calculated following the expression in (30) and the delay is as in (31). Those definitions are derived from [23] and do not require further justification, because the first is distanceagnostic and the second is derived from the throughput and the packet dropping probability through Little's Result,

$$
\begin{gathered}
P_{d r o p, Q}=\tau_{Q} \frac{\left(1-p_{Q}\right) p_{Q}^{R+1}}{1-p_{Q}^{R+1}} \sum_{i=0}^{R}\left(1+\frac{W_{i}-1}{2}\right) \\
D_{Q}=\frac{E[P]^{\prime}}{S_{Q}}\left(1-P_{d r o p, Q}\right)
\end{gathered}
$$

\subsection{PtP link case}

It has been already explained in the first sections why long-distance PtP links are a relevant particular case. Real networks are often based on multi-interface nodes linked to their neighbours through PtP links. For those links, we want to know what performance may be expected as a function of the distance covered.

We have two stations $E_{A}$ and $E_{B}$ that communicate over a distance $d_{A B}$ taking a propagation time $\delta_{A B}=$ $\delta_{B A}=\frac{d_{A B}[K m]}{c[K m / s]}$. In this case, there is full symmetry, so that we have two equations with two unknowns $\tau=$ $\tau_{A}=\tau_{B}$, and $p=p_{A}=p_{B}$. Now (6) is exactly valid, as it is for short distances. For the second equation, we can also perform some simplifications. First, (9) becomes $p=\xi$, and we have

$$
p=\sum_{i=0}^{R} \sum_{j=0}^{C W_{i}} b_{i, j} P\left\{e_{i, j} \longrightarrow e_{i, 0} \text { during } V I_{A B}\right\}
$$

where $K_{j}$ is defined as

$$
K_{j}= \begin{cases}1 & \operatorname{int}\left(N V I_{A B}\right)>j \\ \left(N V I_{A B}-j\right) & \operatorname{int}\left(N V I_{A B}\right)=j \\ 0 & \operatorname{int}\left(N V I_{A B}\right)<j\end{cases}
$$

Second, the steady-state probabilities derived from the Markov chain are no longer station dependent: 


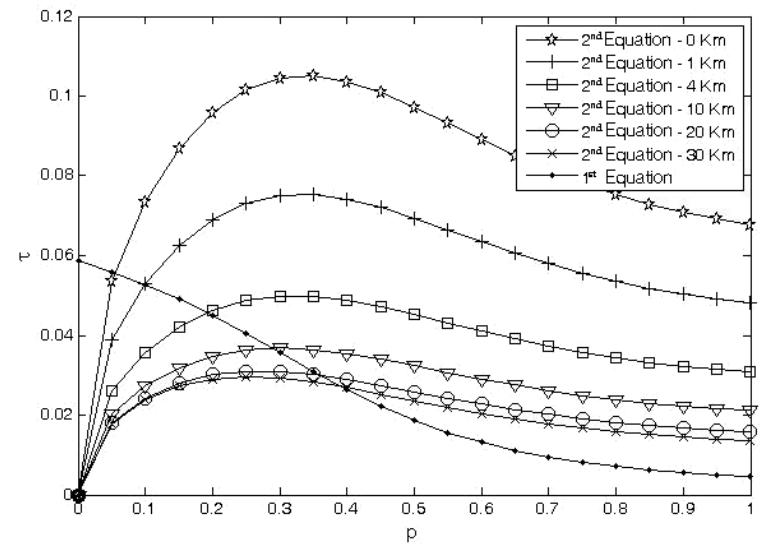

Fig. 6. How the curves representing the PtP model evolve as the distance increases. Equations (6) and (35) are respectively referred to as $1^{\text {st }}$ Equation and $2^{\text {nd }}$ Equation in the legend.

$$
b_{a, b}=\frac{W_{a}-b}{W_{a}} b_{a, 0}=\frac{W_{a}-b}{W_{a}} p^{a} \tau \frac{1-p}{1-p^{R+1}}
$$

and we can also simplify $\mu=1$, and we finally get

$$
\begin{aligned}
p=\sum_{i=0}^{R} \sum_{j=0}^{W_{i}-1} K_{j}\left[\frac { W _ { i } - j } { W _ { i } } p ^ { i } \tau \frac { 1 - p } { 1 - p ^ { R + 1 } } \left(1-\sum_{a=0}^{R}\right.\right. \\
\left.\left.\sum_{b=0}^{W_{a}-1} \min \left(\frac{j}{W_{a}}, 1\right) \frac{W_{a}-b}{W_{a}} p^{a} \tau \frac{1-p}{1-p^{R+1}}\right)\right]
\end{aligned}
$$

We have a system of two equations, (6) and (35), with two unknowns $(p, \tau)$, each of them taking values in the range $[0,1]$. We have checked numerically that the solution is always unique (see Fig. 6), although we have not been able to prove this analytically.

The normalised throughput $S$ can be calculated now with

$$
S=2 \tau \frac{(1-p) E[P]^{\prime}}{E[\text { Slot }]}
$$

where

$$
\begin{array}{rcc}
E[\text { Slot }]= & \left(1-P_{t r}\right) \sigma+P_{t r} P_{s} T_{s}+P_{t r}\left(1-P_{s}\right) T_{c} \\
P_{\text {idle }}= & \left(1-P_{t r}\right)=(1-\tau)^{2} \\
P_{t r} P_{s}= & 2 \tau(1-p) \\
T_{s}= & \frac{E[P]+2 \cdot P L C P+A C K+D I F S+\delta}{1-B_{0}} \\
T_{c}= & E[P]+\text { ACKTimeout }+ \text { DIFS }+\sigma
\end{array}
$$

Packet dropping probability and delay are calculated as

$$
\begin{aligned}
P_{\text {drop }} & =\tau \frac{(1-p) p^{R+1}}{1-p^{R+1}} \sum_{i=0}^{R}\left(1+\frac{W_{i}-1}{2}\right) \\
D & =\frac{2 E[P]^{\prime}}{S}\left(1-P_{\text {drop }}\right)
\end{aligned}
$$

\section{Methods and Materials for Valida- TION}

The model receives as inputs the number of nodes, a distance matrix, and several parameters, such as contention windows, number of retransmissions, slottime and packet size. The result is a triplet composed of the saturation throughput, the average delay and the packet dropping probability for each node. A program named GenSolver has been developed in $\mathrm{C}++$ implementing exactly the proposed model. GenSolver is used to obtain theoretical values of performance at different distances. For PtP links, calculations are done for different link lengths, starting at $0 \mathrm{Km}$ and finishing at $100 \mathrm{Km}$ because longer links are not feasible with CSMA/CA due to the strict limitation of ACKTimeout in the available hardware. For more than two nodes, calculations are obtained for distances up to $40 \mathrm{Km}$ because longer distances do not make sense in PtMP and mesh setups, as shown in Section II. Parameter $\mu_{Q, D}$ in the model fixes the probability that a packet being transmitted by a certain station $E_{Q}$ is intended for another specific station $E_{D}$, and has always been set to $\mu_{Q, D}=\frac{1}{n-1}$ for all $E_{Q}$ and $E_{D}$, which means that each station transmits equally to all others.

The theoretical values were compared with experimental results from three different sources:

- A wireless channel emulator PropSim C8 has been used to obtain experimental results of the performance of IEEE 802.11 point to point links as a function of the propagation time (i.e. the distance). Wireless systems communicating through the emulated channel consisted of embedded computers Soekris Net4511 with a long-range Proxim Orinoco Silver b/g WiFi card, with Voyage Linux as the operating system, and with MadWiFi for controlling the wireless card. Each of the wireless systems were enclosed in an anechoic portable chamber Rhode \& Schwartz CMU-Z10/Z11, in order to guarantee that both systems can see each other only through the channel emulator. The scheme in Fig. 7 represents the whole framework.

Tests were made by injecting traffic with a software tool named iperf. Saturation throughput measurements have been obtained sending bidirectional constant bit rate flows at a rate slightly higher than the maximum for each case.

- As the previous framework only provides a mean for PtP experiments, we also explored network simulators for a better validation. Many network simulators implement the IEEE 802.11 MAC, but some tests run on seven different products showed that only NS-2 [30] and OPNET Modeler [31] seemed to take into account the distance (although the results differed from the experimental observations). NS2 was modified and recompiled in order to enable the adjustment of ACKTimeout and the slot time at simulation time. However, this was not possible 


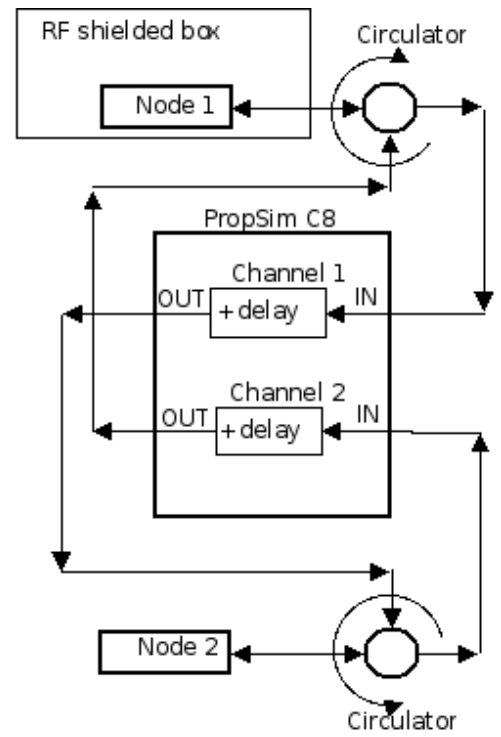

Fig. 7. Schema of long-distance point to point wireless link with PropSim C8 channel emulator.

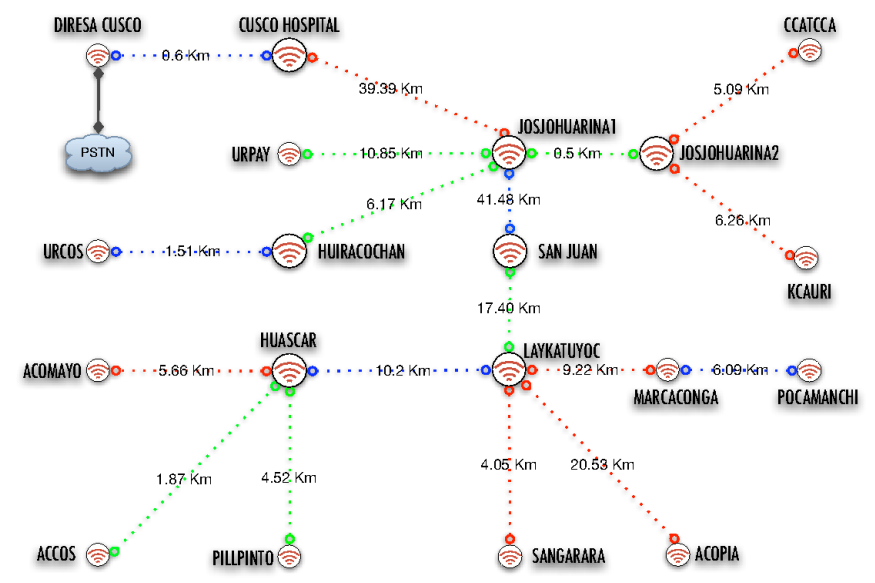

Fig. 8. CuzcoSur network: Network topology and distances among nodes.

with OPNET.

- We had partial access to a real rural WiFi network in Cuzco, Peru, for experimental purposes (see Fig. 8). This network connects rural health centers to the city hospital in Cuzco, Peru, using PtP IEEE 802.11 links for the backbone and PtMP links with no more than 4 nodes in each BSS for the access segments. The network is operational, but it supports no traffic at all during the night. We can run performance tests on the point-to-point links (i.e. any BSS with only 2 active nodes at the moment of the test) using iperf. The 6 links used for the comparisons are those working properly at $2 \mathrm{Mbps}$, covering distances between $1.5 \mathrm{Km}$ and $21 \mathrm{Km}$. Some qualitative tests are also run in the BSS connecting Laykatuyoc, Marcaconga, Sangarara and Acopia, which is the only one with 4 nodes and has only partial visibility among them.
TABLE 1

Parameters for experiments with IEEE 802.11b.

\begin{tabular}{|l|l|}
\hline Nombre del parmetro & Valor \\
\hline$P$ & 8000 bits \\
MACHeader & 224 bits \\
PLCPHeader & 48 bits \\
PLCPPreamble & 144 bits \\
BasicBitRate & $1 \mathrm{Mbps}$ \\
BitRate & $2 \mathrm{Mbps}$ \\
$\delta$ & distance / c \\
ACKTimeout & SIFS $+\sigma_{S T D}+2 \delta+$ PLCP \\
SIFS & $10 \mu s$ \\
$\sigma_{S T D}$ & $20 \mu s$ \\
DIFS & $50 \mu s$ \\
ACK & 112 bits + PLCPHeader + PLCPPreamble \\
$R$ & 7 \\
$C W_{\min }$ & 31 \\
$C W_{\max }$ & 1023 \\
\hline
\end{tabular}

Using the described tools, 1800 traffic injections have been made through the channel emulator at different distances and slot time values (saturating the link for 1 minute each time) and the same number of simulations have been run in NS-2. Additionally, around 150 traffic injections have been performed and measured in the CuzcoSur Network. With these tests, we have measured the evolution of the performance in WiFi links under saturation conditions as the distance among stations changes. All experiments and calculations were based on parameter values given in Table 1 .

The bit rate has always been kept to $2 \mathrm{Mbps}$ for all tests because that is the maximum speed that may be used in all the scenarios and distances considered, and it was also the speed being used in the PtP links at CuzcoSur network at that time.

\section{Model Validation IN STANDARD CONDI- TIONS}

First of all, the theoretical and experimental performances of PtP links for different distances have been compared. In Fig. 9 we compare the theoretical throughput obtained with the model, the experimental results obtained with NS-2 and OPNET simulators, and the experimental results obtained with real 802.11 stations communicating through the channel emulator, all under standard conditions ${ }^{4}$. A discontinuous line shows the prediction by Bianchi \& Tinnirello's model, which gives a reference of the throughput that would be obtained if the distance increased the times, but not the collision probabilities.

We can see in Fig. 9 that the model closely matches the experimental results. The systematic tests run on the channel emulator give very similar values to model predictions, and the measurements on real links from

4. By standard conditions we mean that the slot time, $C W_{\min }$ and other parameters are respected as they are defined in the standard, with the exception of ACKTimeout, that must be modified in order to work over long distances 


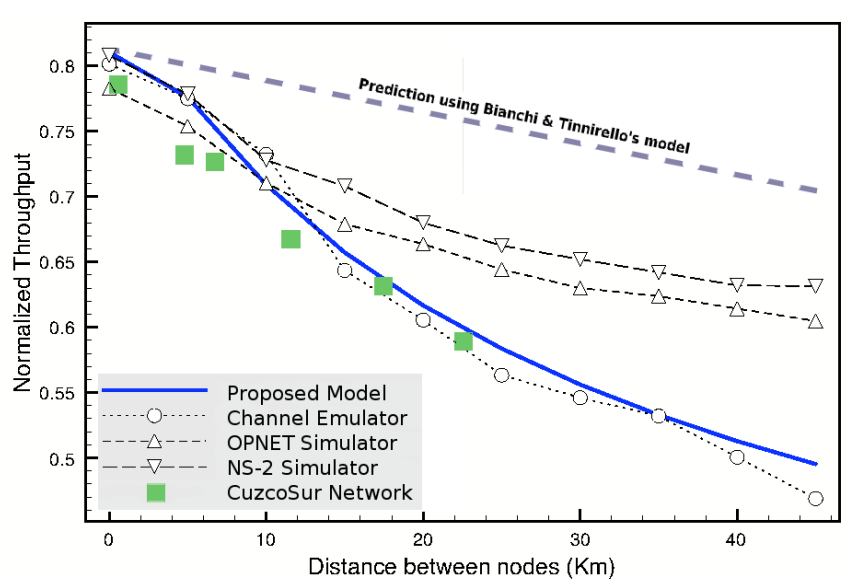

Fig. 9. Normalised througput vs. distance for a point to point link under standard conditions.

TABLE 2

Normalized distances among the 8 nodes used for calculations and simulations.

\begin{tabular}{|c|c|c|c|c|c|c|c|}
\hline & Node 1 & Node 2 & Node 3 & Node 4 & Node 5 & Node 6 & Node 7 \\
\hline \multicolumn{8}{|l|}{ Node 1} \\
\hline Node 2 & 0,25 & & & & & & \\
\hline Node 3 & 0,56 & 0,35 & & & & & \\
\hline Node 4 & 0,56 & 0,35 & 0,5 & & & & \\
\hline Node 5 & 0,76 & 0,52 & 0,28 & 0,45 & & & \\
\hline Node 6 & 0,76 & 0,52 & 0,45 & 0,28 & 0,25 & & \\
\hline Node 7 & 1,03 & 0,79 & 0,5 & 0,71 & 0,28 & 0,45 & \\
\hline Node 8 & 1,03 & 0,79 & 0,71 & 0,5 & 0,45 & 0,28 & 0,5 \\
\hline
\end{tabular}

CuzcoSur network show that the emulated channel is effectively representing the behaviour of real long-distance links very accurately.

On the other hand, NS-2 and OPNET give optimistic results that diverge from experimental results as the distance grows. For the case of NS-2, we have tried to find the reason of this divergence in the simulator source code, but with no conclusive results until now.

For the general case of $n>2$ nodes, we have calculated the theoretical performance for a mesh network with 8 active nodes for which the relative positions have

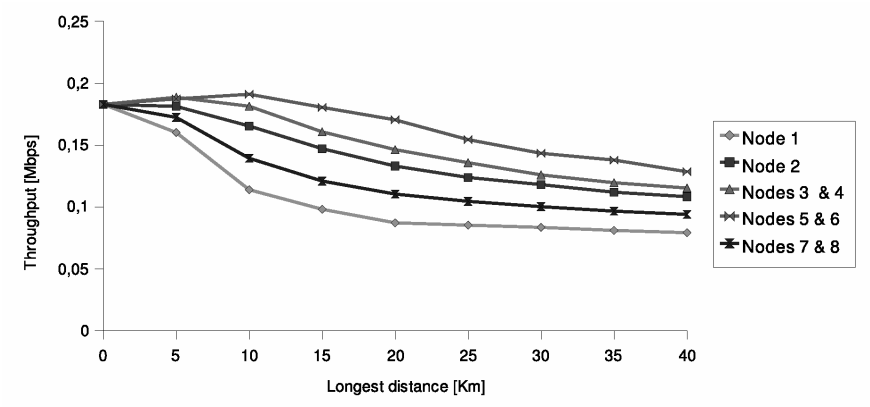

Fig. 10. Saturation throughput obtained with the model for each of 8 nodes at different significant distances.

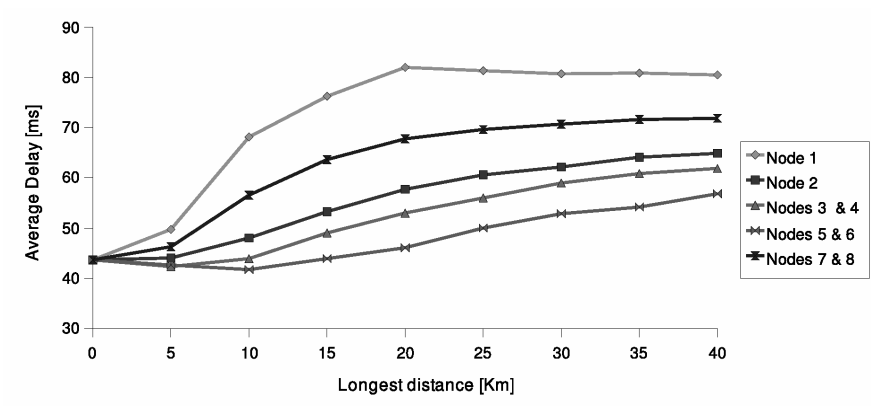

Fig. 11. Average delay obtained with the model for each of 8 nodes at different significant distances.

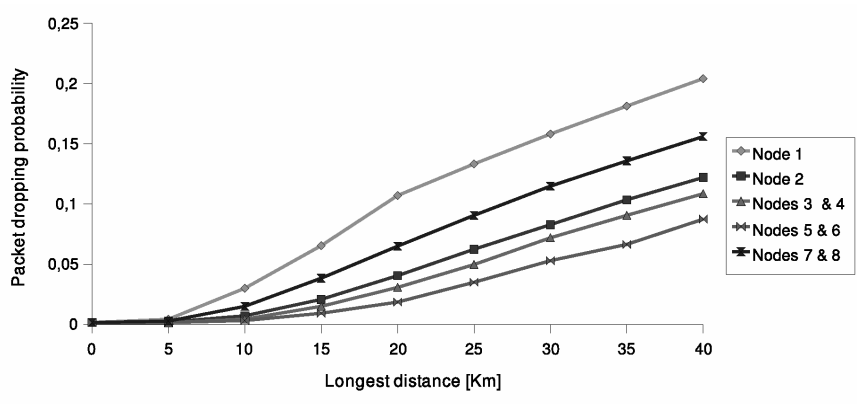

Fig. 12. Packet dropping probability obtained with the model for each of 8 nodes at different significant distances.

been maintained while increasing the absolute distances. The normalized distances among nodes chosen for the study are shown in Table 2. The performance figures have been calculated for different maximum distances from $0 \mathrm{Km}$ up to $40 \mathrm{Km}$ (the maximum distance is between node 1 and node 7 or 8 ), and the results are presented in Figs. 10, 11, and 12 for throughput, delay and packet-drop probability, respectively. The three figures show how different nodes obtain different performance rates due to the different relative distance to the others. This essentially causes a different collision probability that produces unfairness. Different nodes also suffer differently from the impact of the distance on the performance. Finally, we can see the catastrophic effect of the long distances on the packet-drop probability. This is a direct consequence of the high collision probability.

The theoretical results for $n>2$ can not be compared with simulation results because simulators already gave useless results with the PtP case. The channel emulator can only emulate PtP links. Finally, the PtMP cells in CuzcoSur network contain hidden nodes, which makes impossible a quantitative comparison, but experimental measurements demonstrate that different stations get very different performance figures when all contend for the channel in saturation conditions. In fact, the unfairness is much more important than for the case without hidden nodes, some nodes not being able to exchange packets when other more privileged are saturating the BSS. We checked that RTS/CTS does not improve this 
behaviour, as expected.

\section{Performance optimization for long DISTANCES}

The model allows the identification of which parameters could be used to optimize the performance of DCF at long distances. Tuning some of them may give theoretical benefits that are practically useless, like packet size $P$ or maximum number of retransmissions $R$. The packet size impacts on the performance, but there are several considerations at the application level that require some flexibility for that parameter. The number of retransmissions has an ambiguous effect: throughput and delay may be improved by decreasing $R$, but then the number of packets dropped is increased.

The parameters that may be tuned to optimize the performance are slot time and the minimum contention window $W_{0}$. In Eq. (19) we see that the contention windows $W_{i}$ have a direct effect on the final value of collision probabilities $p_{i}$. Also important is the effect of factor $K_{Q D X, j}$, which depends directly on the slot time $(\sigma)$. Hence, we look for the optimum values of $\sigma$ and $W_{i}$ at different distances.

Intuitively, and consistently with the explanations in Section IV.B, reducing the number of backoff slots that may occur during the vulnerability interval reduces the collision probability. The limit will come from the consideration that the slot time contains completely the real round-trip propagation time plus the CCA time, so that two stations really cannot collide unless they start a transmission in the same slot. On the other hand, as the slot time increases, we waste more and more time in the backoff procedure even though the channel may be available, which produces the opposite effect.

Secondly, the increase of maximum contention window size $W_{\min }$ decreases the transmission probability in any given slot, which in turn reduces the collision probability just like increasing the slot time. The advantage of doing so is the possibility of using any 802.11e EDCA compliant products. On the other hand, changing $W_{\min }$ and letting the slot time with the default value adapts the backoff to the distance but keeps the different IFS values unchanged, which may have some negative consequences as well.

A detailed study follows about outlining how performance may be optimized by adjusting one of those two parameters.

\subsection{Optimizing the performance by adjusting $\sigma$}

The variation of the performance with $\sigma$ has been studied using the model, the channel emulator and NS-2 for the PtP case, and then only with the analytical model for $n>2$. The results of throughput versus distance for the PtP case are shown in Fig. 13 for different values of $\sigma$. We can see that in all cases the model approaches the experimental results better than simulations.
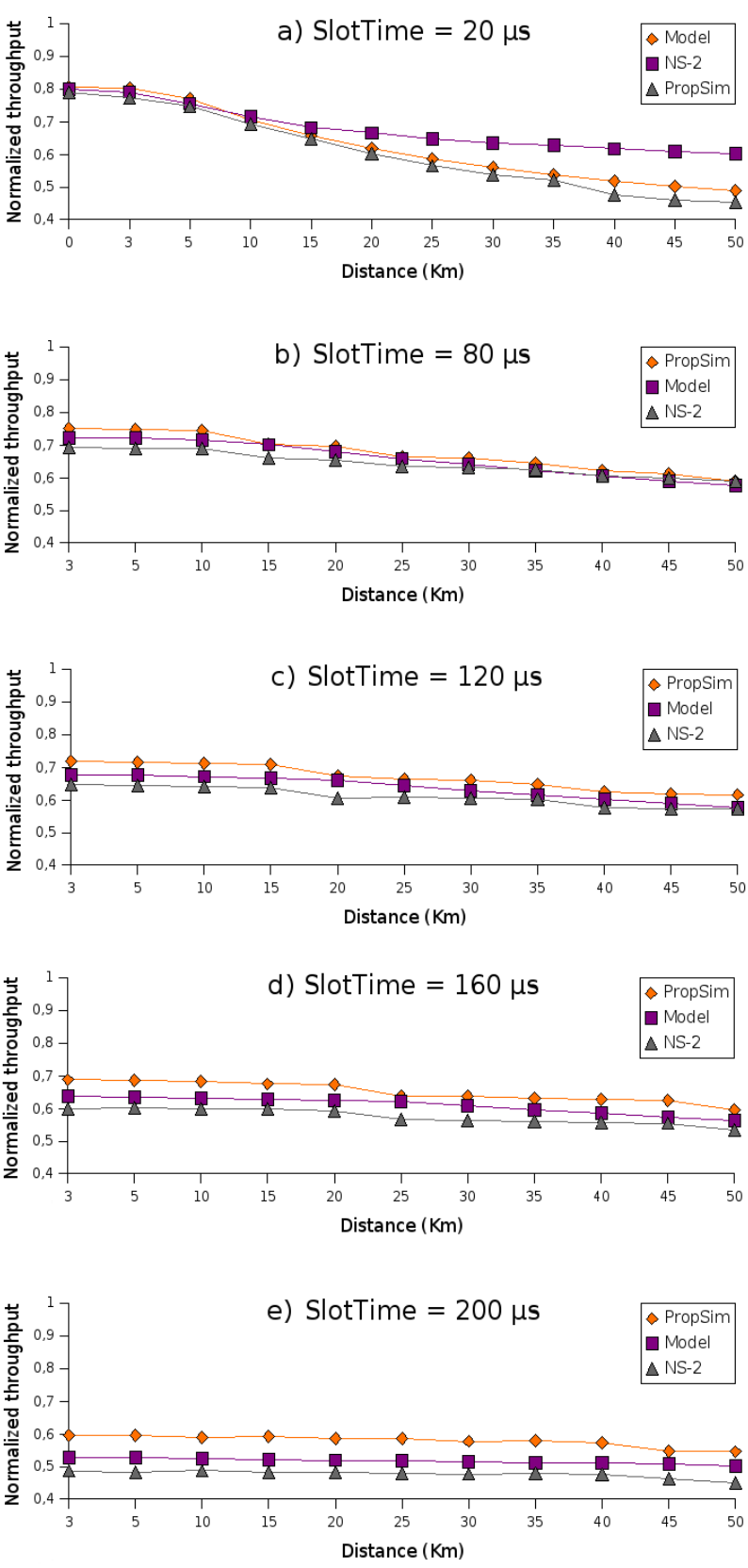

Fig. 13. Normalized througput vs. distance calculated, measured and simulated in a point to point link for a) $\sigma=$ $20 \mu \mathrm{s}, \mathrm{b}) \sigma=80 \mu \mathrm{s}, \mathrm{c}) \sigma=120 \mu \mathrm{s}, \mathrm{d}) \sigma=160 \mu \mathrm{s}$ and e) $\sigma=200 \mu s$.

Figures 14 and 15 present the relationship among throughput, distance and slot time for $n=2$ and for $n=8$ respectively. The figures show how much the throughput can be improved for each distance. For example, we obtain an improvement of $16 \%$ for $n=2$ at $40 \mathrm{Km}$, while the improvement for $n=8$ is $51 \%$ at the same distance. We can also see that the throughput loss due to the time lost in propagation is also much more important for the PtP case $(25 \%$ at $40 \mathrm{Km})$ than for $n=8(12 \%$ at the same distance), but this is due to the fact that we are comparing maximum distances, the 


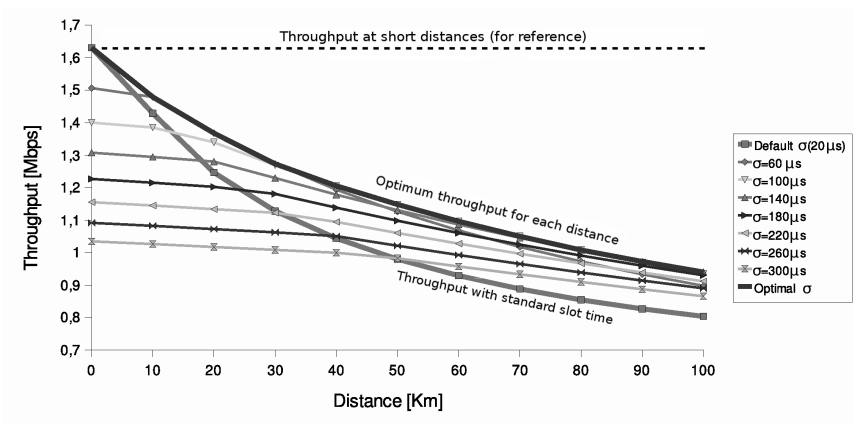

Fig. 14. Throughput vs distance calculated with the model at different slot time values for $n=2$.

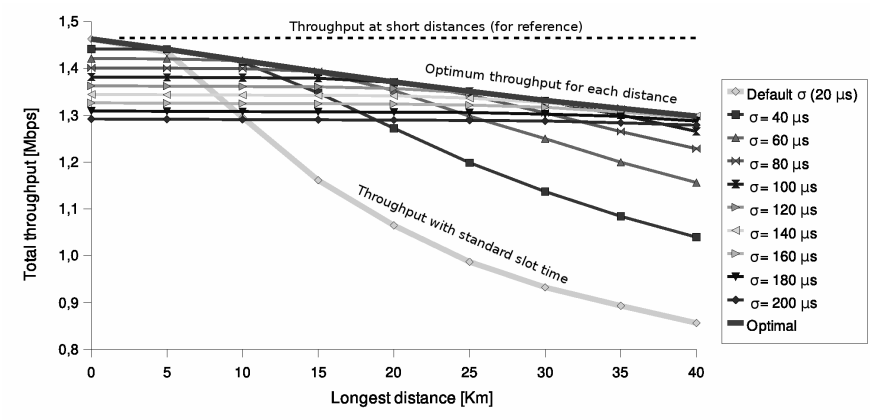

Fig. 15. Throughput vs distance calculated with the model at different slot time values for $n=8$.

difference disappears if we compare average distances.

Figures 16 and 17 present the average delay and packet-drop probability versus distance for different slot times. For $n=8$, Figures 18 and 19 illustrate what happens to each of the eight nodes in terms of delay and the packet loss when we adapt the slot time so that $\sigma \approx \sigma_{S T D}+2 \delta_{M A X}$. We may appreciate that this adjustment of $\sigma$ recovers the long-term fairness of DCF. It can be seen that the values of $\sigma$ maximizing the throughput and minimizing the delay and packet-drop probability are different. Both delay and packet loss obtain optimal values when $\sigma \approx \sigma_{S T D}+2 \delta_{M A X}$, whereas the optimization for the throughput is achieved with $\sigma \leq \sigma_{S T D}+\delta_{M A X}$. This effect on the throughput when the slot time is increased is due to the trade-off between the throughput loss caused by longer contention windows and the throughput gain caused by a reduction in the collision probability. The delay suffers slightly from the increment in the propagation time, but the impact of reducing the collision probability so that most of the frames pass through at the first try is much more important. Finally, the packet dropping probability is independent on the propagation time itself, as it only depends on the collision probability.

The optimization of $\sigma=\sigma_{S T D}+2 \delta_{M A X}$ has many benefits. It is not the best for maximizing the throughput, but it recovers the correct behaviour of DCF. In fact, even RTS/CTS may be used under those conditions because the NAV is not set correctly but the subsequent DIFS

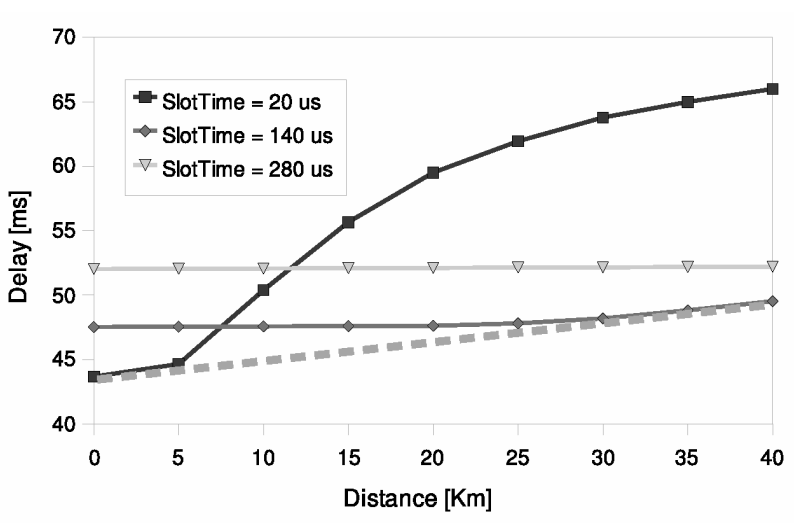

Fig. 16. Average delay vs. distance in a network of 8 nodes calculated with the model for different values of $\sigma$ in saturation conditions.

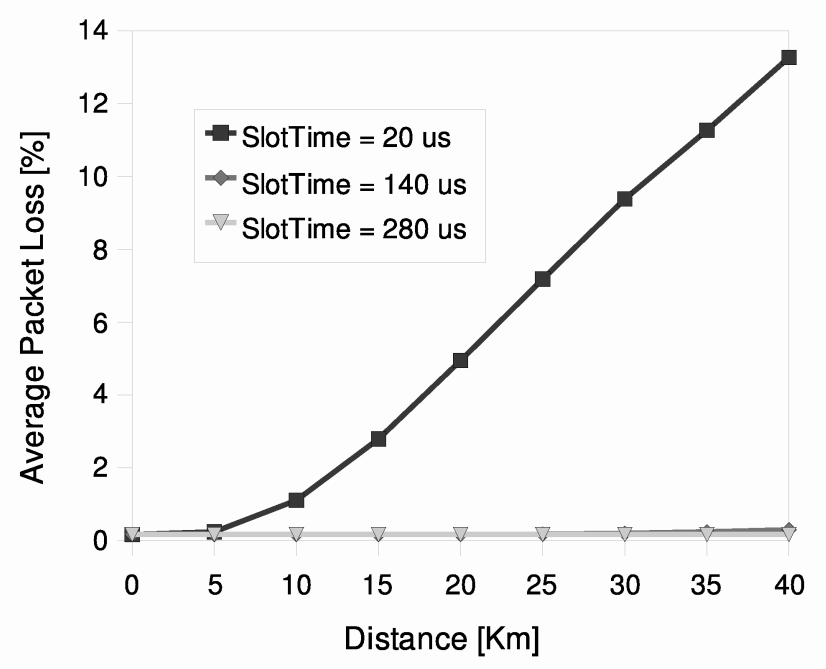

Fig. 17. Packet drop probability for each node vs. distance in a network of 8 nodes calculated with the model for different values of $\sigma$ in saturation conditions.

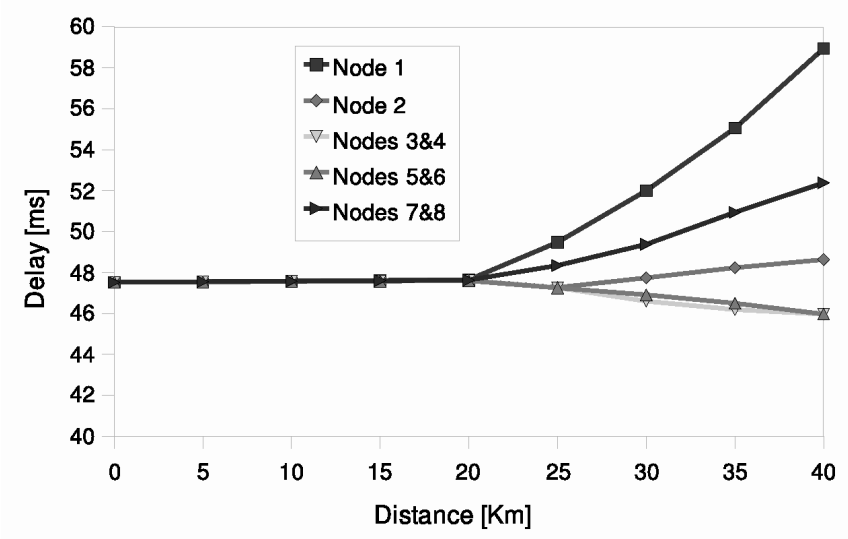

Fig. 18. Average delay for each node vs. distance in a network of 8 nodes calculated with the model for $\sigma=140 \mu \mathrm{s}$. 


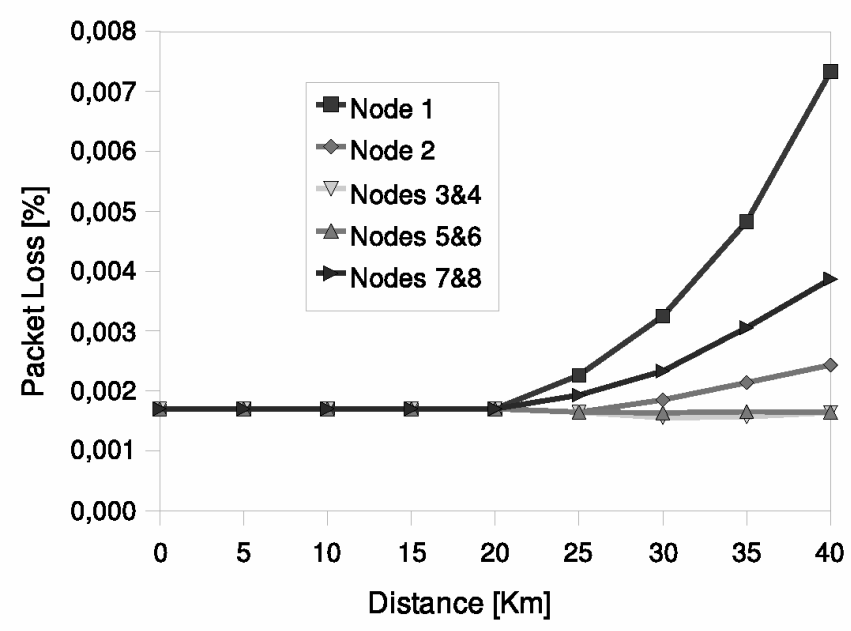

Fig. 19. Packet drop probability for each node vs. distance in a network of 8 nodes calculated with the model for $\sigma=140 \mu s$.

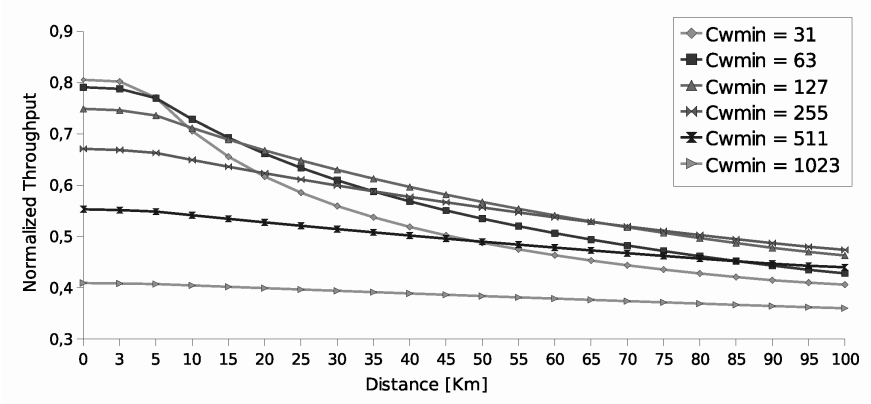

Fig. 20. Throughput versus distance calculated with the model for different values of $C W_{\min }$.

(which includes two adapted slot times) protects the operation against collisions. This has also been qualitatively verified in the PtMP cells of CuzcoSur network.

\subsection{Optimizing the performance with $C W_{\text {min }}$}

Fig. 20 shows the throughput versus distance calculated for different values of $C W_{\min }$. Only values of the form $2^{i}+1$ have been considered. We can see that $C W_{\text {min }}$ may also be used to optimize the throughput. In fact, Fig. 21 shows that the optimal values obtained with both methods are almost the same for all distances. However, increasing the value of $C W_{\min }$ does not modify the DIFS, and then the RTS/CTS mode cannot avoid completely collisions with hidden nodes. This obstacle may be solved in EDCA-compliant systems by adapting both $C W_{\min }$ and AIFS accordingly to the distance, but then those parameters could not be used for differentiating traffic classes.

\section{Conclusions}

In developing countries, there are large isolated rural areas in which IEEE 802.11 DCF can be used for long-

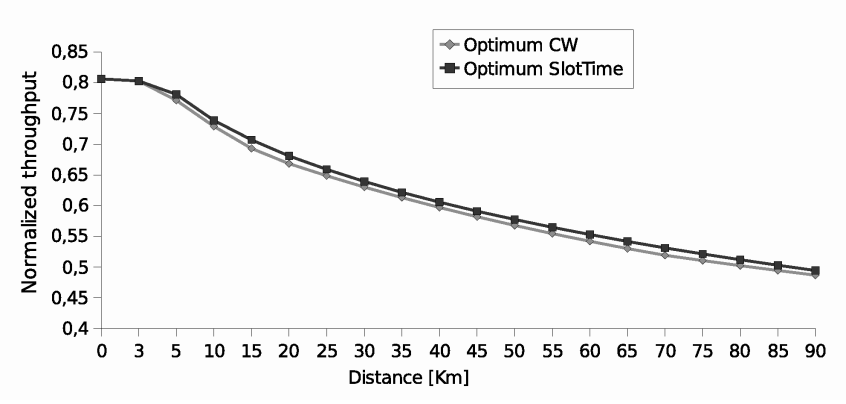

Fig. 21. Comparison between optimization with $C W_{\min }$ and with the slot time $(\sigma)$.

distance low-cost wireless networks. However, there was little formal knowledge concerning the impact of the distance on the performance of the protocol, or about the process of optimizing the performance.

This paper has studied formally the real limits of IEEE 802.11 DCF and has identified the drawbacks for the deployment of long-distance WiFi networks. We have proposed an analytical model of the MAC protocol that accounts for the effect of long propagation times. The model has been used to analyse the performance (in terms of throughput, delay, packet drop probability) and to optimize that performance. It has been shown that a correct adjustment of the ACKTimeout parameter, together with the optimization of the slot time, permit the use of these technologies at very long distances with excellent results. As a golden rule, we obtain a correct behaviour and an acceptable performance in a WiLD network by adding the round-trip propagation time to the default value of both parameters in all stations.

It has also been found that the $C W_{\min }$ parameter may also be used for optimization instead of the slot time, getting similar results. However, in case of the hardware permitting the modification of the slot time, this must be the first choice, because it adapts more integrally the CSMA/CA protocol.

It has also been proved that network simulators implementations of DCF must be improved in order to behave correctly within long distances.

Hence, it has been demonstrated that IEEE 802.11 DCF can be used with slight modifications to the value of the CSMA/CA parameters for wide area rural area networks in developing countries. This is specially true for PtMP cells with maximum distances no longer than $40 \mathrm{Km}$. Longer PtP links are also possible up to $105 \mathrm{Km}$ with systems commercially available, but other solutions not based on CSMA/CA might be more efficient for this case, if available. We permit ourselves to suggest a modification to the IEEE 802.11 standard in the sense of allowing higher values of the air propagation time through the Coverage Class feature. This would be really straight-forward and useful in the context of rural areas at developing countries.

For future works, we will take into account hidden 
nodes and realistic radio channels (with non-null BER) in our analysis. We will also analyse the possibilities offered by IEEE 802.11e for QoS (quality of service) support over long distances, and we will compare the performance obtained with optimized CSMA/CA to other previously proposed alternatives.

\section{ACKNOWLEDGMENTS}

The authors would like to thank Prof. Mark Wilby for his contributions in the development of the GenSolver tool, and also to Prof. Giusseppe Bianchi for his fluid interaction in the discussion of several aspects of his analytical models. We also thank Prof. Jesus Cid for his help in trying to eliminate the condition $\mathrm{H} 4$ of the proposed model.

\section{REFERENCES}

[1] I. 802.11-1999, "Wireless lan medium access control (MAC) and physical layer (PHY) specifications," tech. rep., IEEE Computer Society.

[2] I. 802.11b 1999, "Wireless lan medium access control (MAC) and physical layer (PHY) specifications: Higher-speed physical layer extension in the 2.4 ghz band," tech. rep., IEEE Computer Society.

[3] I. 802.11g 2003, "Wireless lan medium access control (MAC) and physical layer (PHY) specifications: Further higher data rate extension in the 2.4 ghz band," tech. rep., IEEE Computer Society.

[4] I. 802.11-2007, "Wireless lan medium access control (MAC) and physical layer (PHY) specifications, rev. 2007," tech. rep., IEEE Computer Society.

[5] F. J. Proenza, "The road to broadband development in developing countries is through competition driven by wireless and VoIP," in Proc. of Workshop on Wireless Communication and Development: A Global Perspective, (Marina del Rey, California), Oct 2005.

[6] M. Best and R. Roman, "Licence-exempt wireless policies: Unleashing the internet for rural development," in Proc. of Workshop on Wireless Communication and Development: A Global Perspective, (Marina del Rey, California), Oct 2005.

[7] E. Pietrosemoli, "Wireless data transmission in The Andes: Networking Merida state," in Proc. of ISOC INET'99, (San Jose, California), Jun 1999.

[8] A. R. Guntis Barzdins, John Tully, "Applications of high-speed wireless solutions for developing countries: Lessons learned in Latvia and Moldova," in Proc. of ISOC INET'99, (San Jose, California), Jun 1999.

[9] J. Simó-Reigadas, P. Osuna-García, R. Quispe-Tacas, and D. Segundo-Espinoza, "Application of IEEE 802.11 technology for health isolated rural environments," in Proc. of IFIP WCC-WCIT, (Santiago de Chile), August 2006.

[10] P. Bhagwat, B. Raman, and D. Sanghi, "Turning 802.11 insideout," ACM SIGCOMM Computer Communication Review, vol. 34, Jan 2004.

[11] K. Leung, M. Clark, B. McNair, Z. Kostic, L. Cimini, and J. Winters, "Outdoor IEEE 802.11 cellular networks: Radio and mac design and their performance," IEEE Transactions on Vehicular Technology, vol. 56, pp. 2673-2684, Sep 2007.

[12] R. Patra, S. Nedevschi, S. Surana, A. Sheth, L. Subramanian, and E. Brewer, "WiLDNet: Design and implementation of high performance wifi based long distance networks," in Proc. of USENIX NSDI, April 2007.

[13] B. Raman and K. Chebrolu, "Design and evaluation of a new MAC protocol for long-distance 802.11 mesh networks," in Proc. of 11th Annual International Conference on Mobile Computing and Networking, Aug 2005.

[14] S. Salmerón-Ntutumu, J. Simó-Reigadas, and R. Patra, "Comparison of MAC protocols for 802.11-based long distance networks," in Proc. of Workshop Wireless For Development (WIRELESS4D), (Karlstad, Sweden), Dec 2008.
[15] F. Cali, M. Conti, and E. Gregori, "Dynamic tuning of the IEEE 802.11 protocol to achieve a theoretical throughput limit," IEEE/ACM Transactions on Networking, vol. 8, pp. 785-799, Dec 2000.

[16] G. Bianchi, "Performance analysis of the IEEE 802.11 distributed coordination function," IEEE Journal on Selected Areas in Communications, vol. 18, pp. 535-547, Mar 2000.

[17] G. Bianchi and I. Tinnirello, "Remarks on IEEE 802.11 DCF performance analysis," IEEE Communications Letters, vol. 9, pp. 765-767, Aug 2005.

[18] P. Chatzimisios, A. Boucouvalas, and V. Vitsas, "IEEE 802.11 packet delay: a finite retry limit analysis," in Proc. of IEEE Globecom, vol. 2, pp. 950-954, 2003.

[19] X. J. Dong and P. Varaiya, "Saturation throughput analysis of IEEE 802.11 wireless LANs for a lossy channel," IEEE Communications Letters, vol. 9, pp. 100-102, Feb 2005.

[20] J. Simó-Reigadas, P. Osuna-García, S. Lafuente-Sanz, A. MartínezFernández, and J. Seoane-Pascual, "The design of a wireless solar-powered router for rural environments isolated from health facilities," IEEE Wireless Communications, vol. 15, pp. 24-30, Jun 2008.

[21] M. Zennaro, C. Fonda, E. Pietrosemoli, A. S. Okay, R. Flickenger, and S. Radicella, "On a long wireless link for rural telemedicine in Malawi," in 6th Int. Conf. on Open Access, (Lilongwe, Malawi), Nov 2008.

[22] G. Bianchi, "Performance analysis of the IEEE 802.11 distributed coordination function," IEEE Journal on Selected Areas in Communications, vol. 18, pp. 535-547, Mar 2000.

[23] G. Bianchi and I. Tinnirello, "Remarks on IEEE 802.11 DCF performance evaluation," IEEE Communications Letters, vol. 9, pp. 765767, Aug 2005.

[24] G. Bianchi, I. Tinnirello, and L. Scalia, "Understanding 802.11e contention-based prioritization mechanisms and their coexistence with legacy 802.11 stations," IEEE Network, vol. 19, no. 4, pp. 2834, 2005.

[25] J. Simó-Reigadas, C. Figuera-Pozuelo, J. Seoane-Pascual, and A. Martínez-Fernández, "Distance limits in IEEE 802.11 for rural networks in developing countries," in Proc. of IEEE WRECOM, Oct 2007.

[26] "Patch to NS-2.30 for adaptation to long distances." http://download.ehas.org/ns2/2.30.

[27] H. Wu, Y. Peng, K. Long, S. Cheng, , and J. Ma, "Performance of reliable transport protocol over IEEE 802.11 wireless LANs: analysis and enhancement," in Proc. of IEEE INFOCOM, vol. 2, pp. 599-607, 2002.

[28] P. Chatzimisios, A. Boucouvalas, and V. Vitsas, "Throughput and delay analysis of IEEE 802.11 protocol," in Proc. of IEEE International Workshop on Networked Appliances (IWNA), (Liverpool, UK), pp. 168-174, 2002.

[29] H. Chen and Y. Li, "Performance model of IEEE 802.11 DCF with variable packet length," IEEE Communications Letters, vol. 8, pp. 186-188, Mar 2004.

[30] "Network simulator NS-2." http://isi.edu/nsnam/ns.

[31] "OPNET modeler." http://www.opnet.com.

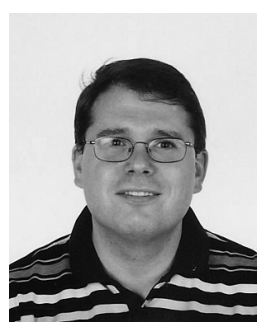

Javier Simó-Reigadas received the B.Sc and Ph.D degrees from the Universidad Politécnica de Madrid, Spain, in 1997 and 2007 repectively. He was a researcher at the EHAS Foundation between 2003 and 2005 in the field of rural broadband networks for developing countries. Since 2005, he is an associate professor with the Department of Signal Theory and Communications at the Universidad Rey Juan Carlos. His main fields of research are broadband wireless technologies for rural regions and strategies for remote network sustainability. 


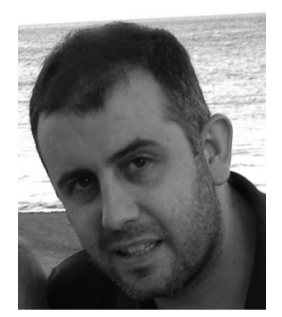

Andrés Martínez-Fernández received the Telecommunication Engineer and the Ph.D. degrees from the Universidad Politécnica, Madrid, Spain, in 1994 and 2003, respectively. $\mathrm{He}$ is currently an Associate Professor in the Department of Theory of Signals and Communications, Universidad Rey Juan Carlos, Madrid. $\mathrm{He}$ is also Director of the EHAS Foundation. His current interests are telemedicine and low-cost telecommunication systems for less developed regions.

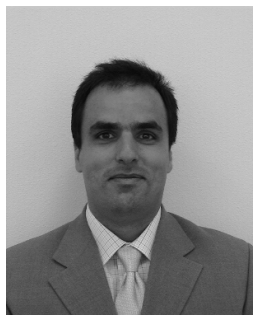

Javier Ramos-López received the B.Sc and M.Sc. degrees from the Universidad Politécnica de Madrid, Spain. Between 1992 and 1995 he cooperated in several research projects at Purdue University, Indiana, USA, working in the field of Signal Processing for Communications. He received the Ph.D degree on 1995 and received the Ericsson award to the best Ph.D. dissertation on Mobile Communications. During 1996 he was Post-Doctoral Research Associate at Purdue University. From 1997 to 2003 Dr Ramos was associate professor at Carlos III University of Madrid. Since $2003 \mathrm{Dr}$ Ramos is the Dean of the Telecommunications Engineering School at the Universidad Rey Juan Carlos. His present fields of research are Broadband Wireless Services and Technologies, Wireless Networks Security and distributed sensing.

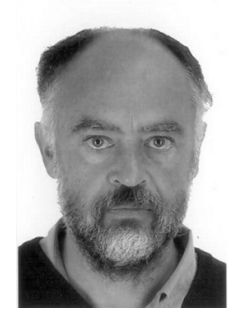

Joaquín Seoane-Pascual got his telecommunication engineering degree in 1976 and a Ph.D in 1989. He worked briefly for real time control and supervision companies, and finally joint the Universidad Politécnica de Madrid in 1978 where he is currently an associate professor in the Telecommunication Engineering School. Since 2000 he also collaborates with the EHAS Foundation in rural telemedicine projects for developing countries. His current interests are distributed systems administration, internationalization and localization, SGML and XML based teaching tools, free software, and low-cost networking for less developed regions. 